\title{
MIXED METHODS FOR DEGENERATE ELLIPTIC PROBLEMS AND APPLICATION TO FRACTIONAL LAPLACIAN
}

\author{
María E. Cejas ${ }^{1, *}$, Ricardo G. Durán ${ }^{2}$ and Mariana I. Prieto ${ }^{3}$
}

\begin{abstract}
We analyze the approximation by mixed finite element methods of solutions of equations of the form $-\operatorname{div}(a \nabla u)=g$, where the coefficient $a=a(x)$ can degenerate going to zero or infinity. First, we extend the classic error analysis to this case provided that the coefficient $a$ belongs to the Muckenhoupt class $A_{2}$. The analysis developed applies to general mixed finite element spaces satisfying the standard commutative diagram property, whenever some stability and interpolation error estimates are valid in weighted norms. Next, we consider in detail the case of Raviart-Thomas spaces of arbitrary order, obtaining optimal order error estimates for simplicial elements in any dimension and for convex quadrilateral elements in the two dimensional case, in both cases under a regularity assumption on the family of meshes. For the lowest order case we show that the regularity assumption can be removed and prove anisotropic error estimates which are of interest in problems with boundary layers. Finally we apply the results to a problem arising in the solution of the fractional Laplace equation.
\end{abstract}

Mathematics Subject Classification. 65N30, 35J70.

Received April 23, 2020. Accepted September 8, 2020.

\section{INTRODUCTION}

In this paper we analyze the approximation by mixed finite element methods of degenerate second order elliptic problems. There is a vast bibliography concerning this kind of methods (see e.g. the books $[7,8]$ and references therein). However, as far as we know, only very few papers have considered the degenerate case (we can mention $[5,28])$.

Let $\mathcal{D} \subset \mathbb{R}^{n}, n \geq 2$, be a bounded Lipschitz polytope and $a \in L_{\text {loc }}^{1}\left(\mathbb{R}^{n}\right)$ be a non-negative function. We assume that the boundary is decomposed into two disjoint parts $\Gamma_{D}$ and $\Gamma_{N}$. Given $g$ and $f$ defined in $\mathcal{D}$ and

Keywords and phrases. Mixed finite elements, degenerate elliptic problems, fractional Laplacian.

1 Centro de Matemática La Plata, Departamento de Matemática, Facultad de Ciencias Exactas, Universidad Nacional de La Plata, Calle 50 y 115, La Plata 1900, Prov. de Buenos Aires, Argentina.

2 IMAS (UBA-CONICET) and Departamento de Matemática, Facultad de Ciencias Exactas y Naturales, Universidad de Buenos Aires, Ciudad Universitaria, Ciudad Autónoma de Buenos Aires 1428, Argentina.

3 Instituto de Matemática (INMABB) - Departamento de Matemática, Universidad Nacional del Sur (UNS)-CONICET, Bahía Blanca, Argentina.

*Corresponding author: mec.eugenia@gmail.com 
on $\Gamma_{N}$ respectively and belonging to appropriate spaces, we consider the problem

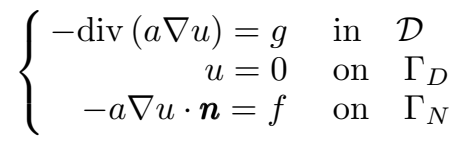

where $\boldsymbol{n}$ denotes the unit exterior normal vector. If $\Gamma_{N}=\partial \mathcal{D}$ we assume the usual compatibility condition $\int_{\mathcal{D}} g=\int_{\partial \mathcal{D}} f$.

We have written the problem in this form in order to simplify notation. However, it is easy to see that all our arguments apply to general problems where the coefficient $a$ is replaced by a matrix $A=A(x)$ satisfying $\lambda a(x)|\xi|^{2} \leq \xi^{T} A(x) \xi \leq \Lambda a(x)|\xi|^{2}$, for all $x \in \mathcal{D}$, where $\lambda$ and $\Lambda$ are positive constants.

We are interested in degenerate problems in the sense that the coefficient $a$ can become infinite or zero in subsets of $\overline{\mathcal{D}}$ with vanishing $n$-dimensional measure. We will assume that $a$ belongs to the Muckenhoupt class $A_{2}$, in particular $a^{-1} \in L^{1}(\mathcal{D})$ and, therefore, the usual mixed method is well defined.

Recall that a non-negative measurable function $a \in L_{\text {loc }}^{1}\left(\mathbb{R}^{n}\right)$ belongs to $A_{2}$ if

$$
[a]_{A_{2}}:=\sup _{Q}\left(\frac{1}{|Q|} \int_{Q} a\right)\left(\frac{1}{|Q|} \int_{Q} a^{-1}\right)<\infty
$$

where the supremum is taken over all cube $Q$ with faces parallel to the coordinate axes.

The class $A_{2}$ was introduced to characterize the weights for which the Hardy-Littlewood maximal operator defined for $f \in L_{\text {loc }}^{1}\left(\mathbb{R}^{n}\right)$ by

$$
\mathcal{M} f(x)=\sup _{r>0} \frac{1}{|B(x, r)|} \int_{|x-y| \leq r}|f(y)| \mathrm{d} y,
$$

is bounded in the associated weighted $L^{2}$-norm (see for instance [12,29]). After that, it was used in the theory of elliptic equations (see e.g. the pioneering work [19]) and, more recently, in the analysis of finite element approximations $[4,30,31]$.

When dealing with anisotropic estimates we will work with the more restrictive strong $A_{2}$ class, which will be denoted by $A_{2}^{s}$ and is defined by

$$
[a]_{A_{2}^{s}}:=\sup _{R}\left(\frac{1}{|R|} \int_{R} a\right)\left(\frac{1}{|R|} \int_{R} a^{-1}\right)<\infty,
$$

where the supremum is taken now over all $n$-dimensional rectangles with faces parallel to the coordinate axes. It is known that $a \in A_{2}^{s}$ if and only if $a$ belongs to $A_{2}$ of one variable for each variable, uniformly in the other variables (see $[21,26]$ ).

Given a weight $a$, we will denote with $L_{a}^{2}(\mathcal{D})$ the usual Hilbert space with measure $a \mathrm{~d} x$. We will also work with the weighted Sobolev space

$$
H_{a}^{1}(\mathcal{D})=\left\{v \in L_{a}^{2}(\mathcal{D}):|\nabla v| \in L_{a}^{2}(\mathcal{D})\right\}
$$

with its natural norm. We recall that $C^{\infty}(\mathcal{D})$ is dense in $H_{a}^{1}(\mathcal{D})$ (see e.g. [25]).

Introducing the variable vector field $\sigma=-a \nabla u$, problem (1.1) can be transformed into the equivalent first order system

$$
\left\{\begin{array}{rll}
\boldsymbol{\sigma}+a \nabla u=0 & \text { in } & \mathcal{D} \\
\operatorname{div} \boldsymbol{\sigma}=g & \text { in } & \mathcal{D} \\
u=0 & \text { on } & \Gamma_{D} \\
\boldsymbol{\sigma} \cdot \boldsymbol{n}=f & \text { on } & \Gamma_{N} .
\end{array}\right.
$$

Then, mixed finite element methods are based on a weak formulation of this system and they approximate simultaneously $\sigma$ and $u$. One motivation for using this type of methods is that, in many applications, the 
variable of physical interest is $\sigma$ and, therefore, it might be more efficient to approximate it directly instead of obtaining it from a computed approximation of $u$. A typical example of this situation is the Darcy equation arising in the simulation of flows in porous media. Indeed, it is many times argued that $\sigma$ is smoother than $\nabla u$. Although this is probably true in practice, it is not possible to give a mathematical foundation to this statement in general (see [20] for an interesting discussion on this subject).

As an application of our results we will consider a problem arising in the solution of the fractional Laplace equation $(-\Delta)^{s} v=f$.

The rest of the paper is organized as follows. In Section 2 we recall the mixed finite element method for (1.1) and extend the classic error analysis to the case of degenerate problems. A fundamental tool is the existence of right inverses of the divergence in weighted norms when the weight belongs to the class $A_{2}$. The analysis given in this section can be applied to general mixed finite element spaces which satisfy the so called commutative diagram property whenever a stability property in a weighted norm for the interpolation operator is valid. Next, in Section 3, we consider the case of Raviart-Thomas elements of arbitrary order on simplicial elements in $\mathbb{R}^{n}$ and prove the stability property mentioned above and optimal order error estimates in weighted norms under the usual regularity assumption on the family of meshes, namely, bounded ratio between outer and inner diameters. At the end of this section we explain how the error estimates can be obtained also for the RaviartThomas approximation using general convex quadrilateral elements in two dimensions, assuming an appropriate regularity assumption. In Section 4 we consider anisotropic error estimates for the Raviart-Thomas spaces of lowest order and prove some weighted interpolation error estimates, where the weights involve the distance to some part of the boundary, for rectangular and prismatic elements. These estimates are of interest in problems with boundary layers. Two important tools in this part of the analysis are the so-called improved Poincaré inequalities and the use of the restricted "Strong $A_{2}$ class". In Section 5, we consider the approximation of the fractional Laplace equation which leads to a particular degenerate problem of the type considered in the previous sections. We show in this example how the weighted error estimates proved for anisotropic elements can be used to design a priori adapted meshes giving almost optimal order with respect to the number of degrees of freedom. Finally, in Section 6 we present some numerical results.

\section{MiXed Finite ELEMEnt APproximations}

To introduce the correct mixed finite element formulation we first analyze the problem in order to know the natural spaces for the original variable $u$ and its associated vector variable $\sigma=-a \nabla u$.

The basic tools for the analysis are the weighted Poincaré inequalities given in the next lemma. The first one is well known while the other is a simple consequence of it.

We will make use of the classic Gagliardo trace theorem, namely, for any Lipschitz domain $\mathcal{D}$ there exists a constant $C$, depending only on $\mathcal{D}$, such that

$$
\|v\|_{L^{1}(\partial \mathcal{D})} \leq C\|v\|_{W^{1,1}(\mathcal{D})} \quad \forall v \in W^{1,1}(\mathcal{D}) .
$$

Observe that if $a \in A_{2}$ we have that

$$
H_{a}^{1}(\mathcal{D}) \subset W^{1,1}(\mathcal{D}),
$$

indeed, $a^{-1} \in L^{1}(\mathcal{D})$ and therefore by the Schwarz inequality we have

$$
\|v\|_{W^{1,1}(\mathcal{D})} \leq\left(\int_{\mathcal{D}} a^{-1}\right)^{1 / 2}\|v\|_{H_{a}^{1}(\mathcal{D})} .
$$

Consequently, traces on $\partial \mathcal{D}$ or on any measurable subset of it are well defined for functions in $H_{a}^{1}(\mathcal{D})$. In particular we can introduce the space

$$
H_{a, \Gamma_{D}}^{1}(\mathcal{D})=\left\{v \in H_{a}^{1}(\mathcal{D}):\left.v\right|_{\Gamma_{D}}=0\right\} .
$$

We denote with $u_{\mathcal{D}}$ and $u_{\Gamma_{D}}$ the averages of $u$ over $\mathcal{D}$ and $\Gamma_{D}$ respectively. In general, along the paper we will write $u_{S}$ for the average of $u$ over a set $S$. 
Lemma 2.1. Let $\mathcal{D}$ be a Lipschitz domain, $\Gamma_{D} \subset \partial \mathcal{D}$ with positive $(n-1)$-measure, and $a \in A_{2}$. Then,

$$
\begin{aligned}
\left\|u-u_{\mathcal{D}}\right\|_{L_{a}^{2}(\mathcal{D})} \leq C\|\nabla u\|_{L_{a}^{2}(\mathcal{D})} & \forall u \in H_{a}^{1}(\mathcal{D}) \\
\left\|u-u_{\Gamma_{D}}\right\|_{L_{a}^{2}(\mathcal{D})} \leq C\|\nabla u\|_{L_{a}^{2}(\mathcal{D})} & \forall u \in H_{a}^{1}(\mathcal{D}),
\end{aligned}
$$

where the constant depends only on $\mathcal{D},\left|\Gamma_{D}\right|$ and $[a]_{A_{2}}$.

Proof. The first one is the well known weighted Poincaré inequality. It was first proved in [19] for the case of a ball and extended for very general domains in several papers (see e.g. $[11,14,24]$ ).

To prove (2.5), taking into account (2.4), it is enough to estimate $\left\|u_{\mathcal{D}}-u_{\Gamma_{D}}\right\|_{L_{a}^{2}(\mathcal{D})}$. But,

$$
\left\|u_{\mathcal{D}}-u_{\Gamma_{D}}\right\|_{L_{a}^{2}(\mathcal{D})}=\left(\int_{\mathcal{D}} a\right)^{1 / 2}\left|u_{\mathcal{D}}-u_{\Gamma_{D}}\right| \leq\left(\int_{\mathcal{D}} a\right)^{1 / 2} \frac{1}{\left|\Gamma_{D}\right|} \int_{\Gamma_{D}}\left|u_{\mathcal{D}}-u\right|,
$$

using (2.1), (2.3), and then (2.4), we have

$$
\begin{aligned}
\int_{\Gamma_{D}}\left|u_{\mathcal{D}}-u\right| & \leq C\left\|u_{\mathcal{D}}-u\right\|_{W^{1,1}(\mathcal{D})} \leq C\left(\int_{\mathcal{D}} a^{-1}\right)^{1 / 2}\left\|u_{\mathcal{D}}-u\right\|_{H_{a}^{1}(\mathcal{D})} \\
& \leq C\left(\int_{\mathcal{D}} a^{-1}\right)^{1 / 2}\|\nabla u\|_{L_{a}^{2}(\mathcal{D})}
\end{aligned}
$$

so that

$$
\left\|u_{\mathcal{D}}-u_{\Gamma_{D}}\right\|_{L_{a}^{2}(\mathcal{D})} \leq C\left(\int_{\mathcal{D}} a\right)^{1 / 2}\left(\int_{\mathcal{D}} a^{-1}\right)^{1 / 2}\|\nabla u\|_{L_{a}^{2}(\mathcal{D})},
$$

and we conclude the proof observing that

$$
\left(\int_{\mathcal{D}} a\right)^{1 / 2}\left(\int_{\mathcal{D}} a^{-1}\right)^{1 / 2} \leq C[a]_{A_{2}}^{1 / 2}
$$

with a constant depending on $\mathcal{D}$.

Consequently, using standard arguments we can prove the well-posedness of problem (1.1).

Theorem 2.2. Given $a \in A_{2}, g \in L_{a^{-1}}^{2}(\mathcal{D})$ and $f \in L^{\infty}\left(\Gamma_{N}\right)$, the problem

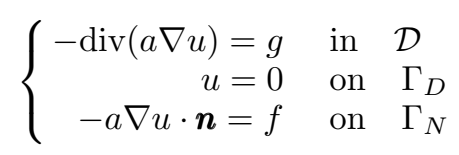

has a unique solution $u \in H_{a}^{1}(\mathcal{D})$, provided $\int_{\mathcal{D}} g=\int_{\partial \mathcal{D}} f$ in the case $\Gamma_{N}=\partial \mathcal{D}$. Moreover,

$$
\|u\|_{H_{a}^{1}(\mathcal{D})} \leq C\left\{\|g\|_{L_{a^{-1}}^{2}(\mathcal{D})}+\|f\|_{L^{\infty}\left(\Gamma_{N}\right)}\right\} \text {. }
$$

Proof. The result follows from standard arguments using the Lax-Milgram theorem. Indeed, considering first the case $\Gamma_{D} \neq \emptyset$, the weak form of the problem is given by: Find $u \in H_{a, \Gamma_{D}}^{1}(\mathcal{D})$ such that

$$
\int_{\mathcal{D}} a \nabla u \cdot \nabla v=\int_{\mathcal{D}} g v-\int_{\Gamma_{N}} f v \quad \forall v \in H_{a, \Gamma_{D}}^{1}(\mathcal{D})
$$

The left hand side is a continuous bilinear form in $H_{a, \Gamma_{D}}^{1}(\mathcal{D})$ and, in view of $(2.5)$, it is coercive. Since $g \in L_{a^{-1}}^{2}(\mathcal{D})$, using again (2.5), it follows that the first term on the right defines a continuous linear form in $H_{a, \Gamma_{D}}^{1}(\mathcal{D})$. Finally, to see that the second term on the right also defines a continuous linear form we use that $f \in L^{\infty}\left(\Gamma_{N}\right)$ and $(2.1)$ combined with the Schwarz inequality.

The case $\Gamma_{N}=\partial \mathcal{D}$ can be treated analogously using now (2.4). 
Remark 2.3. For simplicity we have assumed that $f \in L^{\infty}\left(\Gamma_{N}\right)$. It is clear from the proof that this hypothesis can be relaxed: it is enough that $f$ belong to the dual of the space formed by restrictions to $\Gamma_{N}$ of functions in $H_{a}^{1}(\mathcal{D})$. In the particular case of the application considered in the last section this space is characterized as a fractional Sobolev space.

Let us now introduce the appropriate spaces for the formulation and analysis of mixed approximations. Taking into account Theorem 2.2, the appropriate space for the vector variable $\sigma=-a \nabla u$ is

$$
H_{a^{-1}}(\operatorname{div}, \mathcal{D})=\left\{\boldsymbol{\tau} \in L_{a^{-1}}^{2}(\mathcal{D})^{n}: \operatorname{div} \boldsymbol{\tau} \in L_{a^{-1}}^{2}(\mathcal{D})\right\}
$$

which is a Hilbert space with norm given by

$$
\|\boldsymbol{\tau}\|_{H_{a^{-1}}(\operatorname{div}, \mathcal{D})}^{2}=\|\boldsymbol{\tau}\|_{L_{a^{-1}}^{2}(\mathcal{D})}^{2}+\|\operatorname{div} \boldsymbol{\tau}\|_{L_{a^{-1}}^{2}(\mathcal{D})}^{2} .
$$

To treat Neumann boundary conditions we need to see that the normal component is well defined for any $\tau \in H_{a^{-1}}(\operatorname{div}, \mathcal{D})$ on $\partial \mathcal{D}$ whenever $a \in A_{2}$. It is known (see e.g. [15]) that there exists $q<2$ such that $a^{-1} \in A_{q}$, and therefore, an argument analogous to that used to obtain $(2.2)$ gives that $L_{a^{-1}}^{2}(\mathcal{D}) \subset L^{p}(\mathcal{D})$ for $p=2 / q$. Then,

$$
H_{a^{-1}}(\operatorname{div}, \mathcal{D}) \subset W^{p}(\operatorname{div}, \mathcal{D})=\left\{\boldsymbol{\tau} \in L^{p}(\mathcal{D})^{n}: \operatorname{div} \boldsymbol{\tau} \in L^{p}(\mathcal{D})\right\}
$$

and it is known that, for $\boldsymbol{\tau} \in W^{p}(\operatorname{div}, \mathcal{D}), \boldsymbol{\tau} \cdot \boldsymbol{n}$ is well defined as a distribution that belongs to $W^{-1 / p, p}(\partial \mathcal{D})$.

In the mixed formulation Neumann type boundary conditions are imposed in an essential way, and so we will work with the subspace

$$
H_{a^{-1}, \Gamma_{N}}(\operatorname{div}, \mathcal{D})=\left\{\boldsymbol{\tau} \in H_{a^{-1}}(\operatorname{div}, \mathcal{D}): \boldsymbol{\tau} \cdot \boldsymbol{n}=0 \quad \text { on } \quad \Gamma_{N}\right\}
$$

Dividing by $a$, the first equation in (1.2) can be rewritten as

$$
a^{-1} \boldsymbol{\sigma}+\nabla u=0 \quad \text { in } \mathcal{D},
$$

and multiplying by test functions and integrating by parts, we obtain the weak mixed formulation of problem (1.2), namely, find $\sigma \in H_{a^{-1}}(\operatorname{div}, \mathcal{D})$ and $u \in L_{a}^{2}(\mathcal{D})$ such that

$$
\boldsymbol{\sigma} \cdot \boldsymbol{n}=f \quad \text { on } \quad \Gamma_{N}
$$

and

$$
\left\{\begin{array}{rlrl}
\int_{\mathcal{D}} a^{-1} \boldsymbol{\sigma} \cdot \boldsymbol{\tau}-\int_{\mathcal{D}} u \operatorname{div} \boldsymbol{\tau} & =0 & & \forall \boldsymbol{\tau} \in H_{a^{-1}, \Gamma_{N}}(\operatorname{div}, \mathcal{D}) \\
\int_{\mathcal{D}} v \operatorname{div} \boldsymbol{\sigma}=\int_{\mathcal{D}} g v & \forall v \in L_{a}^{2}(\mathcal{D}) .
\end{array}\right.
$$

Observe that the Dirichlet boundary condition is implicit in the weak formulation. When $\Gamma_{N}=\partial \mathcal{D}, L_{a}^{2}(\mathcal{D})$ has to be replaced by $L_{a, 0}^{2}(\mathcal{D})$, the subspace of functions with vanishing mean value.

As usual, the error analysis is divided in two steps. The first one consists in proving estimates for the finite element approximation error in terms of the error for some appropriate interpolation or projection operator. This part of the analysis can be done for general mixed finite element spaces provided they satisfy the so called commutative diagram property as well as some weighted stability estimates for the appropriate projections. Therefore, we will develop this part of the error analysis for general spaces stating the necessary assumptions that afterwards have to be proved for each particular choice of approximation spaces. The second part consists in estimating the interpolation error. We will do this first for the family of Raviart-Thomas spaces of arbitrary order $k \geq 0$ in simplex and general dimension $n$, and second, for Raviart-Thomas on convex quadrilaterals in dimension 2 (and we will comment on the generalization to the three dimensional case). 
We assume that we have a family of partitions $\left\{\mathcal{T}_{h}\right\}$ of the domain $\mathcal{D}$ such that each $\mathcal{T}_{h}$ is consistent with the boundary conditions, i.e. the exterior boundary of an element is completely contained in $\Gamma_{D}$ or in $\Gamma_{N}$. Associated with these partitions we assume that we have finite element spaces $\boldsymbol{S}_{h} \subset H_{a^{-1}}(\operatorname{div}, \mathcal{D}), V_{h} \subset L_{a}^{2}(\mathcal{D})$ (or $V_{h} \subset L_{a, 0}^{2}(\mathcal{D})$ when $\Gamma_{N}=\partial \mathcal{D}$ ), such that, if

$$
\boldsymbol{S}_{h, N}=\boldsymbol{S}_{h} \cap H_{a^{-1}, \Gamma_{N}}(\operatorname{div}, \mathcal{D}),
$$

then

$$
\operatorname{div} \boldsymbol{S}_{h, N}=V_{h}
$$

and there exists an operator $\Pi_{h}: S \longrightarrow \boldsymbol{S}_{h}$, defined in an appropriate subspace $\boldsymbol{S} \subset H_{a^{-1}}(\operatorname{div}, \mathcal{D})$ containing the solution $\boldsymbol{\sigma}$, such that, if $\boldsymbol{\tau} \in \boldsymbol{S} \cap H_{a^{-1}, \Gamma_{N}}(\operatorname{div}, \mathcal{D})$ then $\Pi_{h} \boldsymbol{\tau} \in \boldsymbol{S}_{h, N}$ and, for all $\boldsymbol{\tau} \in \boldsymbol{S}$,

$$
\int_{\mathcal{D}} \operatorname{div}\left(\boldsymbol{\tau}-\Pi_{h} \boldsymbol{\tau}\right) v=0 \quad \forall v \in V_{h}
$$

Introducing the $L^{2}$-orthogonal projection $P_{h}: L^{2}(\mathcal{D}) \longrightarrow V_{h},(2.9)$ and (2.10) yield the commutative diagram property

$$
\operatorname{div} \Pi_{h}=P_{h} \operatorname{div} .
$$

The mixed finite element approximation of problem (1.2) is given by

$$
\left(\boldsymbol{\sigma}_{h}, u_{h}\right) \in \boldsymbol{S}_{h} \times V_{h}
$$

such that,

$$
\boldsymbol{\sigma}_{h} \cdot \boldsymbol{n}=\Pi_{h} \boldsymbol{\sigma} \cdot \boldsymbol{n} \quad \text { on } \quad \Gamma_{N}
$$

and

$$
\left\{\begin{aligned}
\int_{\mathcal{D}} a^{-1} \boldsymbol{\sigma}_{h} \cdot \boldsymbol{\tau}-\int_{\mathcal{D}} u_{h} \operatorname{div} \boldsymbol{\tau}=0 & \forall \boldsymbol{\tau} \in \boldsymbol{S}_{h, N}, \\
\int_{\mathcal{D}} v \operatorname{div} \boldsymbol{\sigma}_{h},=\int_{\mathcal{D}} g v & \forall v \in V_{h} .
\end{aligned}\right.
$$

Existence and uniqueness of the discrete solution and the following error estimate follow by well known arguments (see e.g. $[7,8]$ ). For completeness we include the proof of the error estimate to show that the usual arguments can be adapted for degenerate problems and for the mixed boundary conditions considered here. We neglect numerical integration errors assuming that all the integrals can be computed exactly.

Lemma 2.4. Assume that $a^{-1} \in L^{1}(\mathcal{D})$ and $\boldsymbol{\sigma} \in L_{a^{-1}}^{2}(\mathcal{D})^{n}$. If $\boldsymbol{\sigma}$ is the solution of (2.7) and (2.8), and $\boldsymbol{\sigma}_{h}$ that of (2.12) and (2.13), then

$$
\left\|\boldsymbol{\sigma}-\boldsymbol{\sigma}_{h}\right\|_{L_{a-1}^{2}} \leq\left\|\boldsymbol{\sigma}-\Pi_{h} \boldsymbol{\sigma}\right\|_{L_{a-1}^{2}} .
$$

Proof. Subtracting the second equation in (2.13) to the second one in (2.8) and using (2.10) we obtain

$$
\int_{\mathcal{D}} \operatorname{div}\left(\Pi_{h} \boldsymbol{\sigma}-\boldsymbol{\sigma}_{h}\right) v=0 \quad \forall v \in V_{h}
$$

From (2.12) it follows that $\Pi_{h} \boldsymbol{\sigma}-\boldsymbol{\sigma}_{h} \in \boldsymbol{S}_{h, N}$, and then, by (2.9) we conclude that $\operatorname{div}\left(\Pi_{h} \boldsymbol{\sigma}-\boldsymbol{\sigma}_{h}\right)=0$. Moreover, taking $\boldsymbol{\tau}=\Pi_{h} \boldsymbol{\sigma}-\boldsymbol{\sigma}_{h}$ in (2.8) and (2.13), we obtain

$$
\int_{\mathcal{D}} a^{-1}\left(\boldsymbol{\sigma}-\boldsymbol{\sigma}_{h}\right) \cdot\left(\Pi_{h} \boldsymbol{\sigma}-\boldsymbol{\sigma}_{h}\right)=0
$$

and so,

and the lemma is proved.

$$
\begin{aligned}
\left\|\boldsymbol{\sigma}-\boldsymbol{\sigma}_{h}\right\|_{L_{a^{2}}^{2}}^{2} & =\int_{\mathcal{D}} a^{-1}\left(\boldsymbol{\sigma}-\boldsymbol{\sigma}_{h}\right) \cdot\left(\boldsymbol{\sigma}-\Pi_{h} \boldsymbol{\sigma}\right) \\
& \leq\left\|\boldsymbol{\sigma}-\boldsymbol{\sigma}_{h}\right\|_{L_{a-1}^{2}}\left\|\boldsymbol{\sigma}-\Pi_{h} \boldsymbol{\sigma}\right\|_{L_{a^{-1}}^{2}}
\end{aligned}
$$


To estimate the error in the approximation of the scalar variable we need the following result which generalizes to the weighted case the existence of continuous right inverses of the divergence.

Lemma 2.5. If $a \in A_{2}$ then, given $\phi \in L_{a^{-1}}^{2}(\mathcal{D})$ (satisfying $\int_{\mathcal{D}} \phi=0$ in the case $\Gamma_{N}=\partial \mathcal{D}$ ), there exists $\tau \in H_{a^{-1}}^{1}(\mathcal{D})^{n} \cap H_{a^{-1}, \Gamma_{N}}(\operatorname{div}, \mathcal{D})$ such that

$$
\operatorname{div} \boldsymbol{\tau}=\phi
$$

and

$$
\|\boldsymbol{\tau}\|_{H_{a-1}^{1}(\mathcal{D})} \leq C\|\phi\|_{L_{a-1}^{2}(\mathcal{D})},
$$

where the constant $C$ depends on $\mathcal{D}$ and a.

Proof. In the case $\Gamma_{N}=\partial \mathcal{D}$ we have $\int_{\mathcal{D}} \phi=0$ and the result is known. Indeed, for domains which are starshaped with respect to a ball it was proved in Theorem 3.1 of [17] and Theorem 1.1 of [33] using Bogovskii's solution of the divergence and the theory of singular integrals. The arguments used there can be extended for the class of John domains using the generalization of Bogovskii's operator introduced in [3] (for more details see also [2]). A different proof was given in Theorem 5.2 of [13] also for the class of John domains.

Suppose now that $\Gamma_{N} \neq \partial \mathcal{D}$. Enlarging the domain in an appropriate way we can obtain a Lipschitz domain $\widetilde{\mathcal{D}}$ such that $\mathcal{D} \varsubsetneqq \widetilde{\mathcal{D}}$ and $\Gamma_{N} \subset \partial \widetilde{\mathcal{D}}$. For example, we can make a smooth deformation of part of $\Gamma_{D}$.

Now, we extend $\phi$ to $\widetilde{\mathcal{D}}$ as

$$
\widetilde{\phi}=\left\{\begin{array}{cl}
\phi(x), & x \in \mathcal{D} \\
-\frac{\int_{\mathcal{D}} \phi}{|\widetilde{\mathcal{D}} \backslash \mathcal{D}|}, & x \in \widetilde{\mathcal{D}} \backslash \mathcal{D}
\end{array}\right.
$$

and then, since $\int_{\widetilde{\mathcal{D}}} \widetilde{\phi} \mathrm{d} x=0$, there exists $\boldsymbol{\tau} \in H_{a^{-1}}^{1}(\widetilde{\mathcal{D}})^{n}$, vanishing on $\partial \widetilde{\mathcal{D}}$ and satisfying

$$
\|\tau\|_{H_{a^{-1}}^{1}(\widetilde{\mathcal{D}})} \leq C\|\widetilde{\phi}\|_{L_{a^{2}-1}(\widetilde{\mathcal{D}})} .
$$

It is easy to see that $\|\widetilde{\phi}\|_{L_{a^{-1}}^{2}(\widetilde{\mathcal{D}})} \leq C\|\phi\|_{L_{a^{-1}}^{2}(\mathcal{D})}$, and, therefore, the restriction of $\boldsymbol{\tau}$ to $\mathcal{D}$ satisfies the required properties.

For the next lemma we need to use the following stability result in a weighted norm:

$$
\left\|\Pi_{h} \boldsymbol{\tau}\right\|_{L_{a^{-1}}^{2}} \leq C\|\boldsymbol{\tau}\|_{H_{a^{-1}}^{1}} .
$$

Assuming that $a \in A_{2}$, we will prove this estimate for the Raviart-Thomas interpolation in a forthcoming section.

Lemma 2.6. Let $(\sigma, u)$ and $\left(\sigma_{h}, u_{h}\right)$ be the solutions of (2.7), (2.8) and (2.12), (2.13) respectively. If $a \in A_{2}$ and $\Pi_{h}$ satisfies (2.14) then

$$
\left\|u-u_{h}\right\|_{L_{a}^{2}} \leq\left\|u-P_{h} u\right\|_{L_{a}^{2}}+C\left\|\sigma-\sigma_{h}\right\|_{L_{a-1}^{2}}
$$

where $C$ depends on the constant in Lemma 2.5.

Proof. Assume first that $\Gamma_{D} \neq \emptyset$. According to Lemma 2.5 there exists $\tau \in H_{a^{-1}}^{1}(\mathcal{D})^{n} \cap H_{a^{-1}, \Gamma_{N}}(\operatorname{div}, \mathcal{D})$ such that

$$
\operatorname{div} \boldsymbol{\tau}=\left(P_{h} u-u_{h}\right) a
$$

and

$$
\|\boldsymbol{\tau}\|_{H_{a-1}^{1}} \leq C\left\|\left(P_{h} u-u_{h}\right) a\right\|_{L_{a-1}^{2}} .
$$


Then,

$$
\begin{aligned}
\left\|P_{h} u-u_{h}\right\|_{L_{a}^{2}}^{2} & =\int_{\mathcal{D}}\left(P_{h} u-u_{h}\right) \operatorname{div} \boldsymbol{\tau}=\int_{\mathcal{D}}\left(P_{h} u-u_{h}\right) \operatorname{div} \Pi_{h} \boldsymbol{\tau} \\
& =\int_{\mathcal{D}}\left(u-u_{h}\right) \operatorname{div} \Pi_{h} \boldsymbol{\tau}=\int_{\mathcal{D}} a^{-1}\left(\boldsymbol{\sigma}-\boldsymbol{\sigma}_{h}\right) \cdot \Pi_{h} \boldsymbol{\tau} \\
& \leq\left\|\boldsymbol{\sigma}-\boldsymbol{\sigma}_{h}\right\|_{L_{a-1}^{2}}\left\|\Pi_{h} \boldsymbol{\tau}\right\|_{L^{2}}^{2} \\
& \leq C\left\|\boldsymbol{\sigma}-\boldsymbol{\sigma}_{h}\right\|_{L_{a-1}^{2}}\|\boldsymbol{\tau}\|_{H_{a-1}^{1}} \\
& \leq C\left\|\boldsymbol{\sigma}-\boldsymbol{\sigma}_{h}\right\|_{L_{a-1}^{2}}\left\|P_{h} u-u_{h}\right\|_{L_{a}^{2}}
\end{aligned}
$$

where we have used (2.10) and (2.14). Then, (2.15) follows by the triangular inequality.

Now, if $\Gamma_{N}=\partial \mathcal{D}$, there exists $\tau \in H_{a^{-1}, \Gamma_{N}}(\operatorname{div}, \mathcal{D})$ such that

$$
\operatorname{div} \boldsymbol{\tau}=\left(P_{h} u-u_{h}\right) a-\overline{\left(P_{h} u-u_{h}\right) a},
$$

where $\overline{\left(P_{h} u-u_{h}\right) a}$ denotes the average of $\left(P_{h} u-u_{h}\right) a$, and

$$
\|\boldsymbol{\tau}\|_{H_{a^{-1}}^{1}} \leq C\left\|\left(P_{h} u-u_{h}\right) a\right\|_{L_{a^{-1}}^{2}} .
$$

Indeed, this follows from Lemma 2.5 and the estimate

$$
\left|\overline{\left(P_{h} u-u_{h}\right) a}\right| \leq \frac{1}{|\mathcal{D}|}\left(\int_{\mathcal{D}} a\right)^{1 / 2}\left\|\left(P_{h} u-u_{h}\right) a\right\|_{L_{a-1}^{2}}
$$

Since $\int_{\mathcal{D}}\left(P_{h} u-u_{h}\right)=0$ we have

$$
\begin{aligned}
\left\|P_{h} u-u_{h}\right\|_{L_{a}^{2}(\mathcal{D})}^{2} & =\int_{\mathcal{D}}\left(P_{h} u-u_{h}\right)\left(\left(P_{h} u-u_{h}\right) a-\overline{\left(P_{h} u-u_{h}\right) a}\right) \\
& =\int_{\mathcal{D}}\left(P_{h} u-u_{h}\right) \operatorname{div} \tau .
\end{aligned}
$$

The rest of the argument follows as in the previous case.

Combining Lemmas 2.4 and 2.6 we obtain the following

Corollary 2.7. Under the same hypotheses of Lemma 2.6 we have

$$
\left\|u-u_{h}\right\|_{L_{a}^{2}} \leq\left\|u-P_{h} u\right\|_{L_{a}^{2}}+C\left\|\boldsymbol{\sigma}-\Pi_{h} \boldsymbol{\sigma}\right\|_{L_{a^{-1}}^{2}} .
$$

\section{Error estimates for RAVIART-Thomas Elements on Simplices}

To apply the results obtained in the previous section we have to prove error estimates for the corresponding operators $\Pi_{h}$ and $P_{h}$. In this section we consider the case of regular partitions made by simplices, namely, if $h_{K}$ and $\rho_{K}$ are the diameters of $K$ and the biggest ball contained in $K$ respectively, we assume that the family of meshes $\left\{\mathcal{T}_{h}\right\}$ satisfy

$$
\frac{h_{K}}{\rho_{K}} \leq \eta
$$

with a constant $\eta$ independent of $h$.

Recall that the local Raviart-Thomas space of order $k \geq 0$ on a simplex $K \subset \mathbb{R}^{n}$ is given by

$$
\mathcal{R} \mathcal{T}_{k}(K)=\mathcal{P}_{k}(K)^{n}+x \mathcal{P}_{k}(K)
$$


where we are using the usual notation $\mathcal{P}_{k}(K)$ to denote the polynomials of degree less than or equal to $k$ restricted to $K$.

Calling $F_{i}$ the faces (edges in $2 \mathrm{~d}$ ) of $K$ and $\boldsymbol{n}_{i}$ the corresponding exterior normal vectors, the Raviart-Thomas interpolation on each element $K$ is defined by (see e.g. [7]),

$$
\int_{F_{i}} \Pi_{K} \boldsymbol{\sigma} \cdot \boldsymbol{n}_{i} p_{k}=\int_{F_{i}} \boldsymbol{\sigma} \cdot \boldsymbol{n}_{i} p_{k} \quad \forall p_{k} \in \mathcal{P}_{k}\left(F_{i}\right), \quad i=1, \ldots, n+1
$$

and, if $k \geq 1$,

$$
\int_{K} \Pi_{K} \boldsymbol{\sigma} \cdot \mathbf{p}_{k-1} \mathrm{~d} x=\int_{K} \boldsymbol{\sigma} \cdot \mathbf{p}_{k-1} \quad \forall \mathbf{p}_{k-1} \in \mathcal{P}_{k-1}^{n}(K) .
$$

Since the restriction of functions in $W^{1,1}(K)$ belong to $L^{1}(\partial K)$, these degrees of freedom are well defined for $\boldsymbol{\sigma} \in W^{1,1}(K)^{n}$. As we already mentioned in Section 2, for $a \in A_{2}, H_{a}^{1}(K) \subset W^{1,1}(K)$ and so $\Pi_{K}$ is well defined in $H_{a}^{1}(K)^{n}$.

Then, the global space for the approximation of the vector variable for a partition $\mathcal{T}_{h}$ is

$$
\boldsymbol{S}_{h}=\left\{\boldsymbol{\tau} \in H_{a^{-1}}(\operatorname{div}, \mathcal{D}):\left.\boldsymbol{\tau}\right|_{K} \in \mathcal{R} \mathcal{T}_{k}(K) \quad \forall K \in \mathcal{T}_{h}\right\}
$$

The associated space for the scalar variable is given by

$$
V_{h}=\left\{v \in V:\left.v\right|_{K} \in \mathcal{P}_{k}(K) \quad \forall K \in \mathcal{T}_{h}\right\},
$$

where $V=L_{a, 0}^{2}(\mathcal{D})$ when $\Gamma_{N}=\partial \mathcal{D}$ or $V=L_{a}^{2}(\mathcal{D})$ otherwise. Then, the projection $P_{h}$ is given locally by $\left.\left(P_{h} v\right)\right|_{K}=P_{K} v$ where $P_{K}: L_{a}^{2}(K) \rightarrow \mathcal{P}_{k}(K)$ is given by

$$
\int_{K}\left(v-P_{K} v\right) p_{k}=0 \quad \forall p_{k} \in \mathcal{P}_{k}(K),
$$

that is, $P_{K}$ is the orthogonal projection in $L^{2}(K)$ (without the weight). We remark that $P_{K}$ is well defined in $L^{1}(K)$ and so, in particular, in $L_{a}^{2}(K)$.

It is not difficult to check that (2.9), (2.10), and consequently (2.11), are satisfied.

To prove error estimates for the Raviart-Thomas interpolation in weighted norms we work first in a fixed reference element $\widehat{K}$ and then change variables using the Piola transform. Given a simplex $K$ let $\Phi$ an affine map given by $\Phi(\hat{x})=A \hat{x}+b$ that transforms $\widehat{K}$ into $K$. The following estimates are well known,

$$
\|A\| \leq \frac{h_{K}}{\rho_{\hat{K}}} \quad \text { and } \quad\left\|A^{-1}\right\| \leq \frac{h_{\hat{K}}}{\rho_{K}}
$$

The Piola transform is given by

$$
\boldsymbol{\sigma}(x)=\frac{1}{|\operatorname{det} A|} A \hat{\boldsymbol{\sigma}}(\hat{x})
$$

where $x=\Phi(\hat{x})$. It is known that (see [7], Lem. 3.4 for details),

$$
\Pi_{\widehat{K}} \hat{\boldsymbol{\sigma}}=\widehat{\Pi_{K} \boldsymbol{\sigma}} .
$$

The next lemma is a generalization to the case of weighted norms of a classic result on polynomial approximation. This result was proved in Sections 4.3 and 4.4 of [31] but we give a shorter proof here. Although we will only work with $L^{2}$ based Sobolev norms, we will write the lemma for the general $L^{p}$ case because the proof is exactly the same. We denote by $W_{a}^{k, p}(\Omega)$ the Banach space of functions with derivatives up to the order $k$ in $L_{a}^{p}(\Omega)$. We refer the reader to [15] for the definition of the $A_{p}$ class of weights as well as for the boundedness of the Hardy-Littlewood maximal operator in weighted norms. 
Suppose that $\Omega \subset \mathbb{R}^{n}$ is star-shaped with respect to a ball $B \subset \Omega$ and let $\varphi=\frac{1}{|B|} \chi_{B}$, where $\chi_{B}$ denotes the characteristic function of $B$. Given an integer number $m \geq 0$ and a function $u \in W^{m, 1}(\Omega)$, the averaged Taylor polynomial of degree less than or equal to $m$ associated with $u \in W^{m, 1}(\Omega)$ is given by

$$
p_{m}(u)(x)=\sum_{|\alpha| \leq m} \frac{1}{\alpha !} \int_{B} D^{\alpha} u(y)(x-y)^{\alpha} \varphi(y) \mathrm{d} y .
$$

In what follows we will use the notation $D^{j} u:=\sum_{|\alpha|=j}\left|D^{\alpha} u\right|$ and extend by zero this function outside $\Omega$.

Lemma 3.1. Let $\Omega \subset \mathbb{R}^{n}$ be a domain with diameter $d$ which is star-shaped with respect to a ball $B$ of radius $\rho$. Then, for $1<p<\infty, m \geq 0$ an integer number, and $u \in W_{a}^{m+1, p}(\Omega)$, where $a \in A_{p}$, the polynomial $p_{m}(u) \in \mathcal{P}_{m}$ satisfies, for $0 \leq j \leq m+1$,

$$
\left\|D^{j}\left(u-p_{m}(u)\right)\right\|_{L_{a}^{p}(\Omega)} \leq C\left(\frac{d}{\rho}\right)^{n} d^{m+1-j}\left\|D^{m+1} u\right\|_{L_{a}^{p}(\Omega)}
$$

with a constant $C$ depending only on $n, m, p$ and $[a]_{A_{p}}$.

Proof. First of all, observe that $p_{m}(u)$ is well defined because $W_{a}^{m+1, p}(\Omega) \subset W^{m+1,1}(\Omega)$. On the other hand, since $D^{\alpha} p_{m}(u)=p_{m-|\alpha|}\left(D^{\alpha} u\right)$ for all $|\alpha| \leq m$ (see [9], Prop. 4.1.17), it is enough to consider the case $j=0$.

According to Proposition 4.2.8 of [9], for any $x \in \Omega$ we have

$$
u(x)-p_{m}(u)(x)=(m+1) \sum_{|\alpha|=m+1} \frac{1}{\alpha !} \int_{0}^{1} \int_{\Omega}(x-z)^{\alpha} \frac{1}{s^{n}} \varphi\left(x+\frac{z-x}{s}\right) D^{\alpha} u(z) \mathrm{d} z \frac{\mathrm{d} s}{s} .
$$

Now, since the integrand vanishes unless $x+\frac{z-x}{s} \in B$ and $x \in \Omega$, we can restrict the set of integration to $\left|x+\frac{z-x}{s}-x\right| \leq d$, or equivalently, to $|z-x| \leq s d$. Therefore, a simple estimate yields

$$
\begin{aligned}
\left|u(x)-p_{m}(u)(x)\right| & \leq C\|\varphi\|_{\infty} d^{n} \int_{0}^{1} \frac{1}{(s d)^{n}} \int_{|z-x| \leq s d}|x-z|^{m+1} D^{m+1} u(z) \mathrm{d} z \frac{\mathrm{d} s}{s} \\
& \leq C\left(\frac{d}{\rho}\right)^{n} \int_{0}^{1} \frac{1}{(s d)^{n}} \int_{|z-x| \leq s d}(s d)^{m+1} D^{m+1} u(z) \mathrm{d} z \frac{\mathrm{d} s}{s} \\
& \leq C\left(\frac{d}{\rho}\right)^{n} d^{m+1} \mathcal{M}\left(D^{m+1} u\right)(x)
\end{aligned}
$$

where for the second inequality we have used that $\|\varphi\|_{\infty} \leq C / \rho^{n}$ while for the third one the definition of the Hardy-Littlewood maximal function. Then, the proof concludes by using the boundedness of the maximal operator in the space $L_{a}^{p}$.

In what follows we will use the following observation: under the regularity assumption (3.1) it is easy to see that

$$
\left(\frac{1}{|K|} \int_{K} a\right)\left(\frac{1}{|K|} \int_{K} a^{-1}\right) \leq C[a]_{A_{2}}
$$

with $C$ depending only on $n$ and $\eta$.

To simplify notation we will prove all the estimates for the weight $a$ although some of them will be used later for $a^{-1}$. Note that, from the definition of $A_{2}$, it follows immediately that $a \in A_{2}$ if and only if $a^{-1} \in A_{2}$.

Theorem 3.2. Given a simplex $K=\Phi(\widehat{K}) \subset \mathbb{R}^{n}$ and $k \geq 0$, let $\Pi_{K}$ be the Raviart-Thomas interpolation over $\mathcal{R} \mathcal{T}_{k}(K)$. Then, if $a \in A_{2}$, there exists a constant $C$ depending only on $n, k,[a]_{A_{2}}$, and the regularity constant $\eta$ such that, for $0 \leq m \leq k$,

$$
\left\|\boldsymbol{\sigma}-\Pi_{K} \boldsymbol{\sigma}\right\|_{L_{a}^{2}(K)} \leq C h_{K}^{m+1}\left\|D^{m+1} \boldsymbol{\sigma}\right\|_{L_{a}^{2}(K)} .
$$


Proof. Proceeding exactly as in Theorem 3.1 of [7], but using here the trace theorem (2.1) on $\hat{K}$, and using (2.3) for $\hat{a}_{K}$ in $\hat{K}$, we obtain

$$
\left|\Pi_{\widehat{K}} \hat{\boldsymbol{\sigma}}(\hat{x})\right| \leq C\|\hat{\boldsymbol{\sigma}}\|_{W^{1,1}(\widehat{K})} \leq C\left(\int_{\widehat{K}} \hat{a}_{K}^{-1}\right)^{1 / 2}\|\hat{\boldsymbol{\sigma}}\|_{H_{\hat{a}_{K}}^{1}(\widehat{K})}
$$

where the constant depends only on the reference element, and so on $n$. Then, taking square in this inequality, multiplying by $\hat{a}_{K}(\hat{x})$, and integrating we get

$$
\left\|\Pi_{\widehat{K}} \hat{\boldsymbol{\sigma}}\right\|_{L_{\hat{a}_{K}}^{2}(\widehat{K})} \leq C\left(\int_{\widehat{K}} \hat{a}_{K}\right)^{1 / 2}\left(\int_{\widehat{K}} \hat{a}_{K}^{-1}\right)^{1 / 2}\|\hat{\boldsymbol{\sigma}}\|_{H_{\hat{a}_{K}}^{1}(\widehat{K})} .
$$

But,

$$
\left(\int_{\widehat{K}} \hat{a}_{K}\right)\left(\int_{\widehat{K}} \hat{a}_{K}^{-1}\right) \leq C\left(\frac{1}{|K|} \int_{K} a\right)\left(\frac{1}{|K|} \int_{K} a^{-1}\right) \leq C[a]_{A_{2}}
$$

where we have used (3.6). Therefore,

$$
\left\|\Pi_{\widehat{K}} \hat{\boldsymbol{\sigma}}\right\|_{L_{\hat{a}_{K}}^{2}(\widehat{K})} \leq C[a]_{A_{2}}^{1 / 2}\|\hat{\boldsymbol{\sigma}}\|_{H_{\hat{a}_{K}}^{1}(\widehat{K})}
$$

where $C$ depends only on $\eta$ and $n$.

Making now the change of variables $x=\Phi(\hat{x})$ and using (3.4) we obtain (see details in [7], Thm. 3.1),

$$
\left\|\Pi_{K} \boldsymbol{\sigma}\right\|_{L_{a}^{2}(K)} \leq C\left\{\|\boldsymbol{\sigma}\|_{L_{a}^{2}(K)}+h_{K}\|D \boldsymbol{\sigma}\|_{L_{a}^{2}(K)}\right\}
$$

where the constant depends only on the reference element, $[a]_{A_{2}}$, and the regularity constant $\eta$.

To conclude the proof we recall that $\mathcal{P}_{k}(K)^{n} \subset \mathcal{R} \mathcal{T}_{k}(K)$ and so, given $\mathbf{p} \in \mathcal{P}_{k}(K)^{n}, \Pi_{K} \mathbf{p}=\mathbf{p}$. Then,

$$
\begin{aligned}
\left\|\boldsymbol{\sigma}-\Pi_{K} \boldsymbol{\sigma}\right\|_{L_{a}^{2}(K)} & =\left\|\boldsymbol{\sigma}-\mathbf{p}-\Pi_{K}(\boldsymbol{\sigma}-\mathbf{p})\right\|_{L_{a}^{2}(K)} \\
& \leq C\left\{\|\boldsymbol{\sigma}-\mathbf{p}\|_{L_{a}^{2}(K)}+h_{K}\|D(\boldsymbol{\sigma}-\mathbf{p})\|_{L_{a}^{2}(K)}\right\}
\end{aligned}
$$

and choosing now, for each $0 \leq m \leq k, \mathbf{p}=\mathbf{p}_{m}(\boldsymbol{\sigma}) \in \mathcal{P}_{m}(K)^{n}$ as in Lemma 3.1, we obtain (3.7).

Lemma 3.3. Given a simplex $K \subset \mathbb{R}^{n}$ and $k \geq 0$, let $P_{K}$ be the $L^{2}$-orthogonal projection over $\mathcal{P}_{k}(K)$. Then, if $a \in A_{2}$, there exists a constant $C$ depending only on $n, k,[a]_{A_{2}}$, and the regularity constant $\eta$ such that,

$$
\left\|P_{K} u\right\|_{L_{a}^{2}(K)} \leq C\|u\|_{L_{a}^{2}(K)}
$$

and, for $0 \leq m \leq k$,

$$
\left\|u-P_{K} u\right\|_{L_{a}^{2}(K)} \leq C h_{K}^{m+1}\left\|D^{m+1} u\right\|_{L_{a}^{2}(K)} .
$$

Proof. Let $\left\{p_{\alpha}\right\}_{|\alpha| \leq k}$ an orthonormal basis of $\mathcal{P}_{k}(K)$. Then we have

$$
P_{K} u(x)=\sum_{\alpha}\left(\int_{K} u p_{\alpha}\right) p_{\alpha}(x)
$$

and then,

$$
\left|P_{K} u(x)\right| \leq \sum_{\alpha}\left\|p_{\alpha}\right\|_{\infty}^{2}\|u\|_{L^{1}(K)} \leq \sum_{\alpha}\left\|p_{\alpha}\right\|_{\infty}^{2}\left(\int_{K} a^{-1}\right)^{1 / 2}\|u\|_{L_{a}^{2}(K)} .
$$

But, since $\left\|p_{\alpha}\right\|_{L^{2}}^{2}=1$, by a standard inverse estimate we know that $\left\|p_{\alpha}\right\|_{\infty}^{2} \leq C /|K|$, and therefore,

$$
\left|P_{K} u(x)\right|^{2} \leq \frac{C}{|K|^{2}}\left(\int_{K} a^{-1}\right)\|u\|_{L_{a}^{2}(K)}^{2}
$$


where the constant depends on $n$ and $k$. Multiplying by $a(x)$, integrating, and using (3.6) we obtain

$$
\left\|P_{K} u\right\|_{L_{a}^{2}(K)}^{2} \leq C[a]_{A_{2}}\|u\|_{L_{a}^{2}(K)}^{2}
$$

an so (3.8) holds.

Now, for any $p \in \mathcal{P}_{m}$ with $0 \leq m \leq k$, we have $P_{K} p=p$, and so

$$
\left\|u-P_{K} u\right\|_{L_{a}^{2}(K)} \leq\left\|u-p-P_{K}(u-p)\right\|_{L_{a}^{2}(K)} \leq C\|u-p\|_{L_{a}^{2}(K)}
$$

where in the last inequality we have used (3.8). Therefore, choosing $p=p_{m}(u)$ as the averaged Taylor polynomial in $K$ given by Lemma 3.1 we obtain (3.9).

Combining the error estimates obtained above with the results of the previous section we can now state the main theorem for approximation by Raviart-Thomas elements on regular families of meshes.

Theorem 3.4. Let $\mathcal{T}_{h}$ be a family of meshes with regularity constant $\eta$ and $h=\max _{K \in \mathcal{T}_{h}} h_{K}$. For $k \geq 0$ let $\boldsymbol{S}_{h}$ and $V_{h}$ the spaces defined in (3.2) and (3.3). If $(\boldsymbol{\sigma}, u)$ and $\left(\boldsymbol{\sigma}_{h}, u_{h}\right) \in \boldsymbol{S}_{h} \times V_{h}$ are the solutions of (2.7) and (2.8), and (2.12) and (2.13) respectively then, for $a \in A_{2}$, there exists a constant $C$ depending only on $\mathcal{D}, a, n$, $k$ and $\eta$ such that, for $0 \leq m \leq k$,

$$
\left\|\boldsymbol{\sigma}-\boldsymbol{\sigma}_{h}\right\|_{L_{a^{-1}}^{2}} \leq C h^{m+1}\left\|D^{m+1} \boldsymbol{\sigma}\right\|_{L_{a^{-1}}^{2}},
$$

and

$$
\left\|u-u_{h}\right\|_{L_{a}^{2}} \leq C h^{m+1}\left\{\left\|D^{m+1} \boldsymbol{\sigma}\right\|_{L_{a^{-1}}^{2}}+\left\|D^{m+1} u\right\|_{L_{a}^{2}}\right\} .
$$

Proof. The error estimate for $\boldsymbol{\sigma}$ follows from Lemma 2.4 combined with the estimate (3.7) applied to the weight $a^{-1}$ (recall that $a \in A_{2}$ if and only if $a^{-1} \in A_{2}$ ).

On the other hand, observe that (3.7) implies the hypothesis (2.14) assumed in Lemma 2.6. Then, to bound the error for $u$ we apply that lemma, (3.7) again, and (3.9).

Similar results can be proved for general quadrilateral elements in two dimensions. Indeed, it is possible to extend the results of [6] to the weighted case. Consider an arbitrary convex quadrilateral $K=F(\widehat{K})$, where $\widehat{K}=[0,1]^{2}$, and $F$ is an invertible bilinear map. Associated with the change of variables $x=F(\hat{x})$ we have the Piola transform given by

$$
\boldsymbol{\sigma}(x)=\mathbf{P}_{F} \hat{\boldsymbol{\sigma}}(\hat{x}):=\left(J_{F}(\hat{x})\right)^{-1} D F(\hat{x}) \hat{\boldsymbol{\sigma}}(\hat{x}),
$$

where $J_{F}$ denotes de Jacobian of $F$. For each $k \geq 0$ the Raviart-Thomas space $\mathcal{R} \mathcal{T}_{k}(K)$ and the associated interpolation are defined first in $\hat{K}$ and then in $K$ via the Piola transform in such a way that $\Pi_{\widehat{K}} \hat{\boldsymbol{\sigma}}=\widehat{\Pi_{K} \boldsymbol{\sigma}}$. We refer the reader to [7] for details.

Following [22] we call $S_{i}, 1 \leq i \leq 4$, the four triangles $S_{i}$ obtained by all possible choices of three vertices of $K$, and $\rho_{S_{i}}$ the diameter of a circle inscribed in $S_{i}$. Then, if $h_{K}$ is the diameter of $K$ and $\rho_{K}=\min _{1 \leq i \leq 4} \rho_{S_{i}}$, the regularity condition is given by $h_{K} / \rho_{K} \leq \eta$.

The arguments and results of [6] can be generalized to weighted norms obtaining, for $a \in A_{2}$ and $0 \leq m \leq k$,

$$
\left\|\boldsymbol{\sigma}-\Pi_{K} \boldsymbol{\sigma}\right\|_{L_{a}^{2}(K)} \leq C h_{K}^{m+1}\left\|D^{m+1} \boldsymbol{\sigma}\right\|_{L_{a}^{2}(K)} .
$$

The general error analysis developed in Section 2 cannot be applied directly because now (2.9) does not hold. However, the arguments given in Section 6 of [6] can be extended to our case to obtain optimal order error estimates for the mixed finite element approximations. 


\section{Anisotropic error estimates}

In this section we restrict ourselves to lowest order elements. Our goal is to prove anisotropic error estimates suitable for problems with boundary layers. For this kind of problems it is useful to have estimates involving a weighted norm on the right hand side where the weight is a power of the distance to some part of the boundary.

To present the main arguments we consider first the case of rectangular elements. Then we show how similar ideas can be applied to prismatic elements which are of interest in the application that we are going to consider in the next section, and more generally, in many problems with solutions presenting boundary layers. The case of simplex can be treated in a similar way but, as in the un-weighted case, anisotropic error estimates are valid only for some particular kind of degenerate elements (see [1]).

\subsection{Rectangular elements}

Recall that the local Raviart-Thomas space of lowest degree for an $n$-dimensional rectangular element

$$
R=\left[a_{1}, b_{1}\right] \times \ldots \times\left[a_{n}, b_{n}\right]
$$

is

$$
\mathcal{R} \mathcal{T}_{0}(R)=\left\{\boldsymbol{\tau}: \boldsymbol{\tau}(x)=\left(\alpha_{1}+\beta_{1} x_{1}, \ldots, \alpha_{n}+\beta_{n} x_{n}\right) \quad \text { with } \quad \alpha_{i}, \beta_{i} \in \mathbb{R}\right\},
$$

while the corresponding local space for the scalar variable is $\mathcal{P}_{0}(R)$.

The Raviart-Thomas interpolation is given locally by

$$
\int_{F} \Pi_{R} \boldsymbol{\tau} \cdot \boldsymbol{n}_{F}=\int_{F} \boldsymbol{\tau} \cdot \boldsymbol{n}_{F}
$$

for all faces $F$ of $R$ where $\boldsymbol{n}_{F}$ denotes a unitary vector normal to $F$. If $P_{R}$ is the $L^{2}$-orthogonal projection over $\mathcal{P}_{0}(R)$, it is not difficult to check that the corresponding global projections satisfy (2.9), (2.10), and consequently (2.11).

We need now the following weighted improved Poincaré inequality, which is well known (see e.g. [14,23]). For $a \in A_{2}$ and $Q$ a cube,

$$
\left\|v-v_{Q}\right\|_{L_{a}^{2}(Q)} \leq C\|d \nabla v\|_{L_{a}^{2}(Q)}
$$

where $d$ denotes the distance to $\partial Q$ and the constant depends on $n$ and $[a]_{A_{2}}$.

If we replace $Q$ by $R$ in the above inequality, it is known that the constant in (4.2) blows up when the ratio between outer and inner diameter goes to infinity. However, we have the following anisotropic version if the weight belongs to the smaller class $A_{2}^{s}$ defined in the introduction. For $i=1, \ldots, n$ we define

$$
d_{i}(x)=\min \left\{\left(b_{i}-x_{i}\right),\left(x_{i}-a_{i}\right)\right\} \quad \text { and } \quad h_{i}=b_{i}-a_{i} .
$$

Lemma 4.1. For $a \in A_{2}^{s}$,

$$
\left\|v-v_{R}\right\|_{L_{a}^{2}(R)} \leq C \sum_{i=1}^{n}\left\|d_{i} \frac{\partial v}{\partial x_{i}}\right\|_{L_{a}^{2}(R)},
$$

with $C$ depending on $n$ and $[a]_{A_{2}^{s}}$.

Proof. We introduce $x_{i}=h_{i} \hat{x}_{i}+a_{i}$ and define $\hat{a}_{R}(\hat{x}):=a(x)$. It follows immediately from (4.2) that, if $Q$ is the unitary cube,

$$
\left\|v-v_{Q}\right\|_{L_{\hat{a}_{R}}^{2}(Q)} \leq C \sum_{i=1}^{n}\left\|d_{i} \frac{\partial v}{\partial x_{i}}\right\|_{L_{\hat{a}_{R}}^{2}(Q)}
$$

where $C$ depends only on $n$ and $\left[\hat{a}_{R}\right]_{A_{2}}$. Then, (4.3) follows by changing variables and using that $\left[\hat{a}_{R}\right]_{A_{2}} \leq C[a]_{A_{2}^{s}}$, which can be easily seen. 
Lemma 4.2. For $a \in A_{2}^{s}$ and $F$ the face contained in $x_{j}=a_{j}$ we have

$$
\left\|v-v_{F}\right\|_{L_{a}^{2}(R)} \leq C\left\{\left\|\left(b_{j}-x_{j}\right) \frac{\partial v}{\partial x_{j}}\right\|_{L_{a}^{2}(R)}+\sum_{i \neq j}\left\|d_{i} \frac{\partial v}{\partial x_{i}}\right\|_{L_{a}^{2}(R)}\right\} .
$$

Proof. By a simple integration by parts in the $x_{j}$ variable we have

$$
\frac{1}{|F|} \int_{F} v \mathrm{~d} S=\frac{1}{|R|} \int_{R} v \mathrm{~d} x+\frac{1}{|R|} \int_{R}\left(x_{j}-b_{j}\right) \frac{\partial v}{\partial x_{j}} \mathrm{~d} x .
$$

Then,

and therefore,

$$
v-v_{F}=v-v_{R}-\frac{1}{|R|} \int_{R}\left(x_{j}-b_{j}\right) \frac{\partial v}{\partial x_{j}} \mathrm{~d} x
$$

$$
\left\|v-v_{F}\right\|_{L_{a}^{2}(R)} \leq\left\|v-v_{R}\right\|_{L_{a}^{2}(R)}+\frac{1}{|R|}\left(\int_{R} a \mathrm{~d} x\right)^{1 / 2} \int_{R}\left(b_{j}-x_{j}\right)\left|\frac{\partial v}{\partial x_{j}}\right| \mathrm{d} x
$$

but, multiplying and dividing by $a^{1 / 2}$ and using the Schwarz inequality we obtain

$$
\int_{R}\left(b_{j}-x_{j}\right)\left|\frac{\partial v}{\partial x_{j}}\right| \mathrm{d} x \leq\left\|\left(b_{j}-x_{j}\right) \frac{\partial v}{\partial x_{j}}\right\|_{L_{a}^{2}(R)}\left(\int_{R} a^{-1} \mathrm{~d} x\right)^{1 / 2}
$$

and consequently,

$$
\left\|v-v_{F}\right\|_{L_{a}^{2}(R)} \leq\left\|v-v_{R}\right\|_{L_{a}^{2}(R)}+[a]_{A_{2}^{s}}^{1 / 2}\left\|\left(b_{j}-x_{j}\right) \frac{\partial v}{\partial x_{j}}\right\|_{L_{a}^{2}(R)} .
$$

Therefore, (4.4) follows from (4.3).

We can now prove anisotropic error estimates for the Raviart-Thomas interpolation $\Pi_{R}$. Observe that each component $\left(\Pi_{R} \boldsymbol{\sigma}\right)_{j}$ depends only on $\boldsymbol{\sigma}_{j}$, and so, to simplify notation we will write simply $\Pi_{R} \boldsymbol{\sigma}_{j}$.

Lemma 4.3. For $a \in A_{2}^{s}$ and $1 \leq j \leq n$,

$$
\left\|\boldsymbol{\sigma}_{j}-\Pi_{R} \boldsymbol{\sigma}_{j}\right\|_{L_{a}^{2}(R)} \leq C\left\{\sum_{i \neq j}\left\|d_{i} \frac{\partial \boldsymbol{\sigma}_{j}}{\partial x_{i}}\right\|_{L_{a}^{2}(R)}+h_{j}\left\|\frac{\partial \boldsymbol{\sigma}_{j}}{\partial x_{j}}\right\|_{L_{a}^{2}(R)}\right\} .
$$

Proof. Since $\boldsymbol{\sigma}_{j}-\Pi_{R} \boldsymbol{\sigma}_{j}$ has vanishing mean value on the face defined by $x_{j}=a_{j}$, we obtain from (4.4)

$$
\begin{aligned}
& \left\|\boldsymbol{\sigma}_{j}-\Pi_{R} \boldsymbol{\sigma}_{j}\right\|_{L_{a}^{2}(R)} \\
& \leq C\left\{\sum_{i \neq j}\left\|d_{i} \frac{\partial}{\partial x_{i}}\left(\boldsymbol{\sigma}_{j}-\Pi_{R} \boldsymbol{\sigma}_{j}\right)\right\|_{L_{a}^{2}(R)}+h_{j}\left\|\frac{\partial}{\partial x_{j}}\left(\boldsymbol{\sigma}_{j}-\Pi_{R} \boldsymbol{\sigma}_{j}\right)\right\|_{L_{a}^{2}(R)}\right\} .
\end{aligned}
$$

But, for $i \neq j, \frac{\partial\left(\Pi_{R} \sigma\right)_{j}}{\partial x_{i}}=0$. On the other hand, from the definition of $\Pi_{R}$ we have

$$
\frac{\partial\left(\Pi_{R} \boldsymbol{\sigma}\right)_{j}}{\partial x_{j}}=P_{R}\left(\frac{\partial \boldsymbol{\sigma}_{j}}{\partial x_{j}}\right)
$$

and a simple argument using the Schwarz inequality shows that, for any $v \in L_{a}^{2}(R)$,

$$
\left\|P_{R} v\right\|_{L_{a}^{2}(R)} \leq[a]_{A_{2}^{s}}^{1 / 2}\|v\|_{L_{a}^{2}(R)}
$$

and therefore,

and the lemma is proved.

$$
\left\|\frac{\partial\left(\Pi_{R} \boldsymbol{\sigma}\right)_{j}}{\partial x_{j}}\right\|_{L_{a}^{2}(R)} \leq[a]_{A_{2}^{s}}^{1 / 2}\left\|\frac{\partial \boldsymbol{\sigma}_{j}}{\partial x_{j}}\right\|_{L_{a}^{2}(R)},
$$




\subsection{Prismatic elements}

For notational convenience we work in $\mathbb{R}^{n+1}$ and introduce the variables $(x, y)$, with $x=\left(x_{1}, \ldots, x_{n}\right) \in \mathbb{R}^{n}$ and $y \in \mathbb{R}$. Therefore, the class $A_{2}^{s}$ denotes now the class of weights satisfying

$$
[a]_{A_{2}^{s}}:=\sup _{R}\left(\frac{1}{|R|} \int_{R} a\right)\left(\frac{1}{|R|} \int_{R} a^{-1}\right)<\infty,
$$

where the supremum is taken over all $n+1$-dimensional rectangles.

We consider elements $P=K \times\left[y_{0}, y_{1}\right]$ where $K$ is an $n$-dimensional simplex and $y_{j} \in \mathbb{R}$ for $j=0,1$.

Similar arguments than those used above for the anisotropic estimates in rectangular elements can be used in this case. To simplify notation, we will prove only the particular weighted estimates that we will need for the application considered in the next section. We will denote by $h_{K}$ the diameter of $K$. The elements considered are anisotropic because no relation between $h_{K}$ and $y_{1}-y_{0}$ is required. On the other hand, for the simplices we assume the regularity condition $h_{K} / \rho_{K} \leq \eta$.

Lemma 4.4. Given $a \in A_{2}^{s}, P=K \times\left[y_{0}, y_{1}\right]$ a prismatic element, and $F_{P}$ a face of $P$ given by $F_{P}:=F \times\left[y_{0}, y_{1}\right]$, where $F$ is a face of $K$, we have

$$
\left\|v-v_{F_{P}}\right\|_{L_{a}^{2}(P)} \leq C\left\{\left\|\left(y-y_{0}\right) \frac{\partial v}{\partial y}\right\|_{L_{a}^{2}(P)}+h_{K}\left\|\nabla_{x} v\right\|_{L_{a}^{2}(P)}\right\} .
$$

Proof. Proceeding as in the proof of (4.3) we can prove the Poincaré type inequality

$$
\left\|v-v_{P}\right\|_{L_{a}^{2}(P)} \leq C\left\{\left\|\left(y-y_{0}\right) \frac{\partial v}{\partial y}\right\|_{L_{a}^{2}(P)}+h_{K}\left\|\nabla_{x} v\right\|_{L_{a}^{2}(P)}\right\} .
$$

We will denote with $\mathrm{d} S_{F}$ and $\mathrm{d} S_{F_{P}}$ the surface measures on $F$ and $F_{P}$ respectively. Calling $x_{F}$ the vertex of $K$ opposite to $F$ and integrating by parts we have

$$
\int_{K}\left(x-x_{F}\right) \cdot \nabla_{x} v \mathrm{~d} x=-n \int_{K} v \mathrm{~d} x+\int_{F}\left(x-x_{F}\right) \cdot \boldsymbol{n}_{F} v \mathrm{~d} S_{F}
$$

but, for $x \in F,\left(x-x_{F}\right) \cdot \boldsymbol{n}_{F}=n|K| /|F|$, and therefore,

$$
\frac{1}{|F|} \int_{F} v \mathrm{~d} S=\frac{1}{|K|} \int_{K} v \mathrm{~d} x+\frac{1}{n|K|} \int_{K}\left(x-x_{F}\right) \cdot \nabla_{x} v \mathrm{~d} x .
$$

Then, integrating in the variable $y$,

$$
\frac{1}{|F|} \int_{F_{P}} v \mathrm{~d} S_{F_{P}}=\frac{1}{|K|} \int_{P} v \mathrm{~d} x \mathrm{~d} y+\frac{1}{n|K|} \int_{P}\left(x-x_{F}\right) \cdot \nabla_{x} v \mathrm{~d} x \mathrm{~d} y
$$

and dividing this equation by $\left(y_{1}-y_{0}\right)$ we obtain

$$
v-v_{F_{P}}=v-v_{P}-\frac{1}{n|P|} \int_{P}\left(x-x_{F}\right) \cdot \nabla_{x} v \mathrm{~d} x \mathrm{~d} y
$$

which, using (4.7) and proceeding as in the last part of the proof of Lemma 4.2, implies (4.6).

Lemma 4.5. Given $a \in A_{2}^{s}, P=K \times\left[y_{0}, y_{1}\right]$ a prismatic element, and $F_{P}$ a face of $P$ given by $F_{P}:=K \times\left\{y_{j}\right\}$, $j=0$ or 1 , we have

$$
\left\|v-v_{F_{P}}\right\|_{L_{a}^{2}(P)} \leq C\left\{\left(y_{1}-y_{0}\right)\left\|\frac{\partial v}{\partial y}\right\|_{L_{a}^{2}(P)}+h_{K}\left\|\nabla_{x} v\right\|_{L_{a}^{2}(P)}\right\} .
$$


Proof. It is analogous to the proof of Lemma 4.2 .

The local Raviart-Thomas space for $P=K \times\left[y_{0}, y_{1}\right]$ is given by

$$
\mathcal{R T}_{0}(P)=\left\{\boldsymbol{\tau}: \boldsymbol{\tau}(x)=\left(a_{1}+b x_{1}, \ldots, a_{n}+b x_{n}, a_{n+1}+c y\right) \quad \text { with } \quad a_{i}, b, c \in \mathbb{R}\right\} .
$$

Given a vector field $\boldsymbol{\sigma}$ we define $\tilde{\boldsymbol{\sigma}}=\left(\sigma_{1}, \ldots, \sigma_{n}\right)$ and write $\boldsymbol{\sigma}=\left(\tilde{\boldsymbol{\sigma}}, \sigma_{n+1}\right)$. Since the normals to the top and bottom faces of $P$ are orthogonal to the other ones, the Raviart-Thomas interpolation can be written as

$$
\Pi_{P} \boldsymbol{\sigma}=\left(\Pi_{K} \tilde{\boldsymbol{\sigma}}, \Pi_{n+1} \sigma_{n+1}\right)
$$

where $\Pi_{K}$ and $\Pi_{n+1}$ depend on $\tilde{\boldsymbol{\sigma}}$ and $\sigma_{n+1}$ respectively. Indeed, they are defined by

$$
\int_{F \times\left[y_{0}, y_{1}\right]} \Pi_{K} \tilde{\boldsymbol{\sigma}} \cdot \boldsymbol{n}_{F}=\int_{F \times\left[y_{0}, y_{1}\right]} \tilde{\boldsymbol{\sigma}} \cdot \boldsymbol{n}_{F}
$$

for all face $F$ of $K$ and

$$
\int_{K \times\left\{y_{j}\right\}} \Pi_{n+1} \sigma_{n+1}=\int_{K \times\left\{y_{j}\right\}} \sigma_{n+1}
$$

for $j=0,1$.

Lemma 4.6. For $a \in A_{2}^{s}$ and $P=K \times\left[y_{0}, y_{1}\right]$, we have

$$
\left\|\tilde{\boldsymbol{\sigma}}-\Pi_{K} \tilde{\boldsymbol{\sigma}}\right\|_{L_{a}^{2}(P)} \leq C\left\{\left\|\left(y-y_{0}\right) \frac{\partial \tilde{\boldsymbol{\sigma}}}{\partial y}\right\|_{L_{a}^{2}(P)}+h_{K}\left\|D_{x} \tilde{\boldsymbol{\sigma}}\right\|_{L_{a}^{2}(P)}\right\}
$$

and

$$
\begin{aligned}
\| \sigma_{n+1} & -\Pi_{n+1} \sigma_{n+1} \|_{L_{a}^{2}(P)} \\
& \leq C\left\{\left(y_{1}-y_{0}\right)\left\|\frac{\partial \sigma_{n+1}}{\partial y}\right\|_{L_{a}^{2}(P)}+h_{K}\left\|\nabla_{x} \sigma_{n+1}\right\|_{L_{a}^{2}(P)}\right\}
\end{aligned}
$$

where $C$ depends only on a and the regularity constant $\eta$.

Proof. Since $\left(\tilde{\boldsymbol{\sigma}}-\Pi_{K} \tilde{\boldsymbol{\sigma}}\right) \cdot \boldsymbol{n}_{F}$ has vanishing mean value on $F_{P}=F \times\left[y_{0}, y_{1}\right]$ we can apply (4.6) to obtain

$$
\begin{aligned}
\|(\tilde{\boldsymbol{\sigma}}- & \left.\Pi_{K} \tilde{\boldsymbol{\sigma}}\right) \cdot \boldsymbol{n}_{F} \|_{L_{a}^{2}(P)} \\
& \left.\leq C\left\{\left\|\left(y-y_{0}\right) \frac{\partial}{\partial y}\left[\left(\tilde{\boldsymbol{\sigma}}-\Pi_{K} \tilde{\boldsymbol{\sigma}}\right) \cdot \boldsymbol{n}_{F}\right]\right\|_{L_{a}^{2}(P)}+h_{K} \| \nabla_{x}\left(\tilde{\boldsymbol{\sigma}}-\Pi_{K} \tilde{\boldsymbol{\sigma}}\right) \cdot \boldsymbol{n}_{F}\right) \|_{L_{a}^{2}(P)}\right\},
\end{aligned}
$$

and using this estimate for $n$ different faces of $K$ together with the regularity assumption, we arrive at

$$
\begin{aligned}
\| \tilde{\boldsymbol{\sigma}}- & \Pi_{K} \tilde{\boldsymbol{\sigma}} \|_{L_{a}^{2}(P)} \\
& \leq C\left\{\left\|\left(y-y_{0}\right) \frac{\partial}{\partial y}\left(\tilde{\boldsymbol{\sigma}}-\Pi_{K} \tilde{\boldsymbol{\sigma}}\right)\right\|_{L_{a}^{2}(P)}+h_{K}\left\|D_{x}\left(\tilde{\boldsymbol{\sigma}}-\Pi_{K} \tilde{\boldsymbol{\sigma}}\right)\right\|_{L_{a}^{2}(P)}\right\} .
\end{aligned}
$$

But $\frac{\partial\left(\Pi_{K} \tilde{\boldsymbol{\sigma}}\right)}{\partial y}=0$ and $\frac{\partial\left(\Pi_{K} \tilde{\boldsymbol{\sigma}}\right)_{i}}{\partial x_{j}}=0$ for $i \neq j$. On the other hand, $\frac{\partial\left(\Pi_{K} \tilde{\boldsymbol{\sigma}}\right)_{i}}{\partial x_{i}}=\frac{\operatorname{div}_{x} \Pi_{K} \tilde{\boldsymbol{\sigma}}}{n}$ and $\operatorname{div}{ }_{x} \Pi_{K} \tilde{\boldsymbol{\sigma}}=\frac{1}{|P|} \int_{P} \operatorname{div}_{x} \tilde{\boldsymbol{\sigma}}$ and so, a simple argument using the Cauchy-Schwarz inequality yields

$$
\left\|\operatorname{div}{ }_{x} \Pi_{K} \tilde{\boldsymbol{\sigma}}\right\|_{L_{a}^{2}(P)} \leq[a]_{A_{2}^{s}}^{1 / 2}\left\|\operatorname{div}{ }_{x} \tilde{\boldsymbol{\sigma}}\right\|_{L_{a}^{2}(P)}
$$

and putting all together we obtain (4.9).

The proof of (4.10) is analogous using now that $\sigma_{n+1}-\Pi_{n+1} \sigma_{n+1}$ has vanishing mean value on the face $K \times\left\{y_{0}\right\}$, applying (4.8), and using that $\nabla_{x}\left(\Pi_{n+1} \sigma_{n+1}\right)=0$ and $\frac{\partial}{\partial y}\left(\Pi_{n+1} \sigma_{n+1}\right)=\frac{1}{|P|} \int_{P} \frac{\partial \sigma_{n+1}}{\partial y}$. 


\section{Fractional Laplacian}

As an interesting application of the general results for degenerate problems we consider the spectral fractional Laplacian equation. Given $\Omega \subset \mathbb{R}^{n}$ and $f \in \mathbb{H}^{-s}(\Omega)$ (see the definition of this space below) we want to solve

$$
\left\{\begin{array}{rll}
(-\Delta)^{s} v=f & \text { in } & \Omega \\
v=0 & \text { in } & \partial \Omega
\end{array}\right.
$$

for $0<s<1$.

Caffarelli and Silvestre [10] have shown that the solution of this problem can be obtained as $v(x)=u(x, 0)$ where $u(x, y)$ is the solution of a degenerate elliptic problem, as those considered in the previous sections, in a cylindrical domain in $n+1$ variables, with $a\left(x_{1}, \ldots, x_{n}, y\right)=|y|^{\alpha}$, namely,

$$
\left\{\begin{array}{rrr}
\operatorname{div}\left(y^{\alpha} \nabla u(x, y)\right)=0 & \text { in } & \mathcal{C}=\Omega \times(0, \infty) \\
-\lim _{y \rightarrow 0} y^{\alpha} \frac{\partial u}{\partial y}=d_{s} f & \text { on } & \Gamma_{N}=\Omega \times\{0\} \\
u=0 & \text { on } & \Gamma_{D}=\partial \mathcal{C} \backslash \Gamma_{N}
\end{array}\right.
$$

with $d_{s}=2^{1-2 s} \frac{\Gamma(1-s)}{\Gamma(s)}$ and $\alpha=1-2 s$. To solve this equation numerically one has to approximate the domain $\mathcal{C}$ by a bounded one. With this goal we consider a problem analogous to $(5.2)$ with $\mathcal{C}$ replaced by $\mathcal{C}_{L}=\Omega \times(0, L)$ and adding a homogeneous Dirichlet boundary condition on the upper boundary of $\mathcal{C}_{L}$, namely, we look for $u_{L}$ such that,

$$
\left\{\begin{array}{rll}
\operatorname{div}\left(y^{\alpha} \nabla u_{L}(x, y)\right)=0 & \text { in } & \mathcal{C}_{L}=\Omega \times(0, L) \\
-\lim _{y \rightarrow 0} y^{\alpha} \frac{\partial u_{L}}{\partial y}=d_{s} f & \text { on } & \Gamma_{N}=\Omega \times\{0\} \\
u_{L}=0 & \text { on } & \Gamma_{D}=\partial \mathcal{C}_{L} \backslash \Gamma_{N} .
\end{array}\right.
$$

We will use several results proved in [30], therefore, we recall some notation used in that paper. For $0<s<1$, we denote $H^{s}(\Omega)$ the fractional Sobolev space of order $s$. We define for $s \neq \frac{1}{2}, \mathbb{H}^{s}(\Omega):=H_{0}^{s}(\Omega)$, the closure of $C_{0}^{\infty}(\Omega)$ in $H^{s}(\Omega)$ and $\mathbb{H}^{1 / 2}(\Omega):=H_{00}^{1 / 2}(\Omega)$, the interpolation space $\left[H_{0}^{1}(\Omega), L^{2}(\Omega)\right]_{1 / 2}$ obtained by the K-method (for details see $[27]$ ). $\mathbb{H}^{-s}(\Omega)$ denotes the dual space of $\mathbb{H}^{s}(\Omega)$ for $s \in(0,1)$.

For our error estimates we will need some a priori bounds for the derivatives of the exact solution.

Assuming that $\Omega$ is convex, in [30] the following a priori estimates for the solution of problem (5.2) were proved,

and, for $1 \leq i, j \leq n$,

$$
\|\nabla u\|_{L_{y^{\alpha}}^{2}(\mathcal{C})} \leq C\|f\|_{\mathbb{H}^{-s}(\Omega)}
$$

$$
\left\|\frac{\partial^{2} u}{\partial x_{i} \partial x_{j}}\right\|_{L_{y^{\alpha}}^{2}(\mathcal{C})}+\left\|\frac{\partial^{2} u}{\partial x_{i} \partial y}\right\|_{L_{y^{\alpha}}^{2}(\mathcal{C})} \leq C\|f\|_{\mathbb{H}^{1-s}(\Omega)} .
$$

We will use the following estimate: for $\gamma>-1$ and $v \in L^{1}\left(\mathcal{C}_{L}\right)$ such that $\int_{\mathcal{C}_{L}} v=0$ and $y|\nabla v| \in L_{y^{\gamma}}^{2}\left(\mathcal{C}_{L}\right)$, there exists a constant $C$ independent of $L$ such that

$$
\|v\|_{L_{y \gamma}^{2}\left(\mathcal{C}_{L}\right)} \leq C\|y \nabla v\|_{L_{y \gamma}^{2}\left(\mathcal{C}_{L}\right)} .
$$

This estimate can be proved using the arguments introduced in [14]. Details of the proof are given in Lemma 2.2 of [18] for a square domain but the arguments apply to more general domains, in particular to the cylindrical ones considered here. That the constant $C$ does not depend on $L$ follows from the case $L=1$ combined with a standard scaling argument.

Lemma 5.1. Let $u$ be the solution of (5.2) and $\boldsymbol{\sigma}=\left(\sigma_{1}, \ldots, \sigma_{n+1}\right)=-y^{\alpha} \nabla u$. Then, for $1 \leq j \leq n$ and $1 \leq i \leq n+1$,

$$
\left\|\frac{\partial \sigma_{i}}{\partial x_{j}}\right\|_{L_{y^{-\alpha}}^{2}\left(\mathcal{C}_{L}\right)}+\left\|\frac{\partial \sigma_{n+1}}{\partial y}\right\|_{L_{y^{-\alpha}}^{2}\left(\mathcal{C}_{L}\right)} \leq C\|f\|_{\mathbb{H}^{1-s}(\Omega)},
$$


and for $1 \leq i \leq n$ and $\beta>1-\alpha$,

$$
\left\|\frac{\partial \sigma_{i}}{\partial y}\right\|_{L_{y^{-\alpha+\beta}}^{2}\left(\mathcal{C}_{L}\right)} \leq C L^{\beta / 2}\|f\|_{\mathbb{H}^{1-s}(\Omega)} .
$$

Proof. The bound for the first term in (5.7) follows immediately from (5.5). To estimate the second term observe that, from (5.2),

$$
\frac{\partial \sigma_{n+1}}{\partial y}=-y^{\alpha} \Delta_{x} u
$$

and use (5.5).

For $1 \leq i \leq n$ we have

$$
\frac{\partial \sigma_{i}}{\partial y}=-\alpha y^{\alpha-1} \frac{\partial u}{\partial x_{i}}-y^{\alpha} \frac{\partial^{2} u}{\partial x_{i} \partial y}
$$

To bound the second term we use again (5.5). For the first one we observe that $\int_{\mathcal{C}_{L}} \frac{\partial u}{\partial x_{i}}=0$ because $u$ vanishes on $\partial \Omega \times(0, \infty)$, and therefore, since $\beta>1-\alpha$ we can use (5.6) with $\gamma=\alpha-2+\beta$ to obtain

$$
\begin{aligned}
\int_{\mathcal{C}_{L}}\left|y^{\alpha-1} \frac{\partial u}{\partial x_{i}}\right|^{2} y^{-\alpha} y^{\beta} & =\int_{\mathcal{C}_{L}}\left|\frac{\partial u}{\partial x_{i}}\right|^{2} y^{\alpha-2+\beta} \leq C \int_{\mathcal{C}_{L}}\left|\nabla\left(\frac{\partial u}{\partial x_{i}}\right)\right|^{2} y^{\alpha+\beta} \\
& \leq C L^{\beta} \int_{\mathcal{C}_{L}}\left|\nabla\left(\frac{\partial u}{\partial x_{i}}\right)\right|^{2} y^{\alpha} \leq C L^{\beta}\|f\|_{\mathbb{H}^{1-s}(\Omega)}^{2}
\end{aligned}
$$

where we have used (5.5) for the last inequality.

Our goal is to approximate $u$ and $\boldsymbol{\sigma}=-y^{\alpha} \nabla u$ given by (5.2). Since the problem is posed in the unbounded domain $\mathcal{C}$ we need to replace it by $\mathcal{C}_{L}$ where $L$ will be chosen in terms of the mesh parameter $h$ in such a way that $L \rightarrow \infty$ when $h \rightarrow 0$.

It was shown in Theorem 3.5 of [30] that for $f \in \mathbb{H}^{-s}(\Omega)$ and $L \geq 1$, if $u_{L}(x, y)$ is extended by zero for $y>L$, there exists a constant $C$ such that

$$
\left\|\nabla\left(u-u_{L}\right)\right\|_{L_{y^{\alpha}}^{2}(\mathcal{C})} \leq C e^{-\sqrt{\lambda_{1}} L / 4}\|f\|_{\mathbb{H}^{-s}(\Omega)}
$$

where $\lambda_{1}>0$ is the first eigenvalue of the Laplacian with Dirichlet boundary conditions in $\Omega$.

Moreover, using the Poincaré inequality

$$
\left\|u-u_{L}\right\|_{L_{y^{\alpha}}^{2}(\mathcal{C})} \leq C\left\|\nabla\left(u-u_{L}\right)\right\|_{L_{y^{\alpha}}^{2}(\mathcal{C})},
$$

which follows easily applying the standard Poincaré inequality in $\Omega$ for each $y$, multiplying by the weight, and integrating in $y$, we also have

$$
\left\|u-u_{L}\right\|_{H_{y^{\alpha}}^{1}(\mathcal{C})} \leq C e^{-\sqrt{\lambda_{1}} L / 4}\|f\|_{\mathbb{H}^{-s}(\Omega)} .
$$

Now we consider the mixed finite element approximation of (5.3). We will apply the results of the previous sections for $\mathcal{D}=\mathcal{C}_{L}$ and $\Gamma_{N}=\Omega \times\{0\}$. However, since we want error estimates in terms of $\boldsymbol{\sigma}$ instead of $\boldsymbol{\sigma}_{L}$, to take advantage of the known a priori estimates, we need to introduce some minor modifications in the error analysis.

Given a family of meshes $\mathcal{T}_{h}$ made by prismatic elements as those considered in the last part of Section 4 and the associated spaces $\boldsymbol{S}_{h} \subset H_{y^{-\alpha}}\left(\operatorname{div}, \mathcal{C}_{L}\right)$ and $V_{h} \subset L_{y^{\alpha}}^{2}\left(\mathcal{C}_{L}\right)$ defined locally by $\mathcal{R} \mathcal{T}_{0}(P)$ and $\mathcal{P}_{0}(P)$ respectively, the approximate solutions $u_{L, h} \in V_{h}$ and $\boldsymbol{\sigma}_{L, h} \in \boldsymbol{S}_{h}$ are given by,

$$
\left.\boldsymbol{\sigma}_{L, h} \cdot \boldsymbol{n}\right|_{F}=\frac{d_{s}}{|F|} \int_{F} f,
$$


for every face $F$ contained in $\Omega$, and

$$
\left\{\begin{array}{rlrl}
\int_{\mathcal{C}_{L}} y^{-\alpha} \boldsymbol{\sigma}_{L, h} \cdot \boldsymbol{\tau}-\int_{\mathcal{C}_{L}} u_{L, h} \operatorname{div} \boldsymbol{\tau}=0 & & \forall \boldsymbol{\tau} \in \boldsymbol{S}_{h, N} \\
\int_{\mathcal{C}_{L}} v \operatorname{div} \boldsymbol{\sigma}_{L, h}=0 & \forall v \in V_{h}
\end{array}\right.
$$

where $\boldsymbol{S}_{h, N}:=\boldsymbol{S}_{h} \cap H_{y^{-\alpha}, \Gamma_{N}}\left(\operatorname{div}, \mathcal{C}_{L}\right)$.

Theorem 5.2. Let $u$ and $u_{L}$ be the solutions of (5.2) and (5.3) respectively, $\boldsymbol{\sigma}=-y^{\alpha} \nabla u$ and $\boldsymbol{\sigma}_{L}=-y^{\alpha} \nabla u_{L}$. If $u_{L, h}$ and $\boldsymbol{\sigma}_{L, h}$ are the approximate solutions given by (5.13), then

$$
\left\|\boldsymbol{\sigma}-\boldsymbol{\sigma}_{L, h}\right\|_{L_{y^{-\alpha}}^{2}\left(\mathcal{C}_{L}\right)} \leq\left\|\boldsymbol{\sigma}-\Pi_{h} \boldsymbol{\sigma}\right\|_{L_{y^{-\alpha}}^{2}\left(\mathcal{C}_{L}\right)}+2\left\|\boldsymbol{\sigma}-\boldsymbol{\sigma}_{L}\right\|_{L_{y^{-\alpha}}^{2}\left(\mathcal{C}_{L}\right)},
$$

and

$$
\begin{aligned}
\left\|u-u_{L, h}\right\|_{L_{y}^{2}\left(\mathcal{C}_{L}\right)} \leq & C\left\|u-P_{h} u\right\|_{L_{y^{\alpha}}^{2}\left(\mathcal{C}_{L}\right)} \\
& +C L\left\{\left\|\boldsymbol{\sigma}-\Pi_{h} \boldsymbol{\sigma}\right\|_{L_{y^{-\alpha}}^{2}\left(\mathcal{C}_{L}\right)}+\left\|\boldsymbol{\sigma}-\boldsymbol{\sigma}_{L}\right\|_{L_{y^{-\alpha}}^{2}\left(\mathcal{C}_{L}\right)}\right\} .
\end{aligned}
$$

Proof. Observing that $\Pi_{h} \boldsymbol{\sigma}-\boldsymbol{\sigma}_{L, h} \in \boldsymbol{S}_{h, N}$ and $\operatorname{div}\left(\Pi_{h} \boldsymbol{\sigma}-\boldsymbol{\sigma}_{L, h}\right)=0$ and proceeding as in the proof of Lemma 2.4 we obtain

$$
\int_{\mathcal{C}_{L}} y^{-\alpha}\left(\boldsymbol{\sigma}_{L}-\boldsymbol{\sigma}_{L, h}\right) \cdot\left(\Pi_{h} \boldsymbol{\sigma}-\boldsymbol{\sigma}_{L, h}\right)=0 .
$$

Then,

$$
\left\|\boldsymbol{\sigma}_{L}-\boldsymbol{\sigma}_{L, h}\right\|_{L^{2}-\alpha}^{2}\left(\mathcal{C}_{L}\right)=\int_{\mathcal{C}_{L}} y^{-\alpha}\left(\boldsymbol{\sigma}_{L}-\boldsymbol{\sigma}_{L, h}\right) \cdot\left(\boldsymbol{\sigma}_{L}-\Pi_{h} \boldsymbol{\sigma}\right)
$$

and therefore,

$$
\left\|\boldsymbol{\sigma}_{L}-\boldsymbol{\sigma}_{L, h}\right\|_{L_{y^{-\alpha}}\left(\mathcal{C}_{L}\right)} \leq\left\|\boldsymbol{\sigma}_{L}-\Pi_{h} \boldsymbol{\sigma}\right\|_{L_{y^{-\alpha}}\left(\mathcal{C}_{L}\right)},
$$

which combined with a triangular inequality yields (5.14).

On the other hand, for our domain $\mathcal{C}_{L}$ the inequality from Lemma 2.6 can be written as

$$
\left\|u_{L}-u_{L, h}\right\|_{L_{y^{\alpha}}^{2}\left(\mathcal{C}_{L}\right)} \leq\left\|u_{L}-P_{h} u_{L}\right\|_{L_{y^{\alpha}}^{2}\left(\mathcal{C}_{L}\right)}+C L\left\|\boldsymbol{\sigma}_{L}-\sigma_{L, h}\right\|_{L_{y^{-\alpha}}^{2}\left(\mathcal{C}_{L}\right)}
$$

where the constant $C$ is independent of $L$. Indeed, this follows from the proof of that lemma once we know that the constant in Lemma 2.5 is proportional to $L$, which follows from the case $L=1$ and a scaling argument.

To bound the second term in the right hand side of (5.17) we use (5.16), while for the first one we have

$$
\begin{aligned}
\left\|u_{L}-P_{h} u_{L}\right\|_{L_{y^{\alpha}}^{2}\left(\mathcal{C}_{L}\right)} & \leq\left\|u-P_{h} u\right\|_{L_{y^{\alpha}}^{2}\left(\mathcal{C}_{L}\right)}+\left\|\left(u-u_{L}\right)-P_{h}\left(u-u_{L}\right)\right\|_{L_{y^{\alpha}}^{2}\left(\mathcal{C}_{L}\right)} \\
& \leq\left\|u-P_{h} u\right\|_{L_{y^{\alpha}}^{2}\left(\mathcal{C}_{L}\right)}+C\left\|\nabla\left(u-u_{L}\right)\right\|_{L_{y^{\alpha}}^{2}\left(\mathcal{C}_{L}\right)}
\end{aligned}
$$

where in the last inequality we have used the version for prisms of (4.3). To conclude the proof we observe that

$$
\left\|\nabla\left(u-u_{L}\right)\right\|_{L_{y^{\alpha}}^{2}\left(\mathcal{C}_{L}\right)}=\left\|\boldsymbol{\sigma}-\boldsymbol{\sigma}_{L}\right\|_{L_{y^{-\alpha}}^{2}\left(\mathcal{C}_{L}\right)}
$$

and, therefore, from the Poincaré inequality (5.10) we obtain

$$
\left\|u-u_{L}\right\|_{L_{y^{\alpha}}^{2}\left(\mathcal{C}_{L}\right)} \leq C\left\|\boldsymbol{\sigma}-\boldsymbol{\sigma}_{L}\right\|_{L_{y}^{2}-\alpha}\left(\mathcal{C}_{L}\right) .
$$


Next we are going to show that introducing appropriate meshes, graded in the $y$-direction, we obtain almost optimal order of convergence with respect to the number of nodes, i.e. the same order than that valid for problems with smooth solutions using uniform meshes, up to a logarithmic factor.

Given a mesh-size $h>0$, to define $\mathcal{T}_{h}$ we start with a quasi-uniform triangulation of $\Omega$ made of simplices of diameter less than or equal to $h$. Then, for $L \geq 1$ to be chosen below in terms of $h$, we introduce a partition of $[0, L]$ given by

$$
y_{j}=\left(\frac{j}{N}\right)^{\frac{2}{2-\beta}} L, \quad j=0, \ldots, N
$$

where $N \sim 1 / h$ (we take $N=1 / h$ if it is an integer or some approximation of it if not), and $\beta \in(1-\alpha, 2)$ to be chosen (in the numerical experiments we have taken $\beta$ as the midpoint of this interval). Finally, the partition $\mathcal{T}_{h}$ of $\mathcal{C}_{L}$ is formed by the prismatic elements $P=K \times\left[y_{j}, y_{j+1}\right]$, where $K$ are the elements in the partition of $\Omega$.

It follows from this definition that, for $j \geq 1$,

$$
\left(y_{j+1}-y_{j}\right)^{2} \leq C_{\beta} h^{2} y_{j}^{\beta} L^{2-\beta},
$$

indeed, by the mean value theorem and using that $h \sim 1 / N$ we have

$$
y_{j+1}-y_{j} \leq C \frac{\beta}{2-\beta}(j h)^{\frac{\beta}{2-\beta}} h L \leq C \frac{\beta}{2-\beta} y_{j}^{\frac{\beta}{2}} h L^{1-\frac{\beta}{2}} .
$$

Using the notation introduced for prismatic elements in the previous section, the Raviart-Thomas interpolation is given by $\Pi_{h} \boldsymbol{\sigma}=\left(\tilde{\Pi}_{h} \tilde{\boldsymbol{\sigma}}, \Pi_{h, n+1} \sigma_{n+1}\right)$ where $\tilde{\Pi}_{h}$ and $\Pi_{h, n+1}$ are given locally by $\Pi_{K}$ and $\Pi_{n+1}$ respectively. We recall that, since $-1<\alpha<1, y^{\alpha}$ and $y^{-\alpha}$ belong to $A_{2}^{s}$.

Theorem 5.3. For some $\beta \in(1-\alpha, 2)$, consider the family of meshes $\mathcal{T}_{h}$ defined above. Let $u$ be the solution of (5.2), $\boldsymbol{\sigma}=-y^{\alpha} \nabla u$, and $\left(u_{L, h}, \boldsymbol{\sigma}_{L, h}\right)$ be the approximation given by (5.12) and (5.13). Then, if $L=C_{1}|\log h|$ with $C_{1} \geq 4 / \sqrt{\lambda_{1}}$, we have

$$
\left\|\boldsymbol{\sigma}-\boldsymbol{\sigma}_{L, h}\right\|_{L^{2}-\alpha}\left(\mathcal{C}_{L}\right) \leq C h|\log h|\|f\|_{\mathbb{H}^{1-s}(\Omega)}
$$

and

$$
\left\|u-u_{L, h}\right\|_{L_{y^{\alpha}}^{2}\left(\mathcal{C}_{L}\right)} \leq C h|\log h|^{2}\|f\|_{\mathbb{H}^{1-s}(\Omega)},
$$

where the constant $C$ depends on $\Omega, \alpha$, and $\beta$.

Proof. From (5.14) and (5.9) we have

$$
\left\|\boldsymbol{\sigma}-\boldsymbol{\sigma}_{L, h}\right\|_{L_{y^{-\alpha}}^{2}\left(\mathcal{C}_{L}\right)} \leq\left\|\boldsymbol{\sigma}-\Pi_{h} \boldsymbol{\sigma}\right\|_{L_{y^{-\alpha}}^{2}\left(\mathcal{C}_{L}\right)}+C e^{-\sqrt{\lambda_{1}} L / 4}\|f\|_{\mathbb{H}^{-s}(\Omega)}
$$

Applying (4.9) for the elements of the form $P=K \times\left[0, y_{1}\right]$ and summing over all of them we obtain,

$$
\left\|\tilde{\boldsymbol{\sigma}}-\tilde{\Pi}_{h} \tilde{\boldsymbol{\sigma}}\right\|_{L_{y^{-\alpha}}^{2}\left(\Omega \times\left[0, y_{1}\right]\right)}^{2} \leq C\left\{h^{2}\left\|D_{x} \tilde{\boldsymbol{\sigma}}\right\|_{L_{y^{-\alpha}}^{2}\left(\Omega \times\left[0, y_{1}\right]\right)}^{2}+\left\|y \frac{\partial \tilde{\boldsymbol{\sigma}}}{\partial y}\right\|_{L_{y^{-\alpha}}^{2}\left(\Omega \times\left[0, y_{1}\right]\right)}^{2}\right\} .
$$

But,

$$
\begin{aligned}
\left\|y \frac{\partial \tilde{\boldsymbol{\sigma}}}{\partial y}\right\|_{L_{y^{-\alpha}}^{2}\left(\Omega \times\left[0, y_{1}\right]\right)}^{2} & =\int_{\Omega} \int_{0}^{y_{1}} y^{2}\left|\frac{\partial \tilde{\boldsymbol{\sigma}}}{\partial y}\right|^{2} y^{-\alpha} \mathrm{d} y \mathrm{~d} x \\
& \leq y_{1}^{2-\beta} \int_{\Omega} \int_{0}^{y_{1}}\left|\frac{\partial \tilde{\boldsymbol{\sigma}}}{\partial y}\right|^{2} y^{-\alpha+\beta} \mathrm{d} y \mathrm{~d} x \\
& \leq C h^{2} L^{2-\beta} \int_{\Omega} \int_{0}^{y_{1}}\left|\frac{\partial \tilde{\boldsymbol{\sigma}}}{\partial y}\right|^{2} y^{-\alpha+\beta} \mathrm{d} y \mathrm{~d} x
\end{aligned}
$$


where in the last inequality we have used the definition of $y_{1}$. Then,

$$
\begin{aligned}
& \left\|\tilde{\boldsymbol{\sigma}}-\tilde{\Pi}_{h} \tilde{\boldsymbol{\sigma}}\right\|_{L^{-\alpha}\left(\Omega \times\left[0, y_{1}\right]\right)}^{2} \\
& \quad \leq C\left\{h^{2}\left\|D_{x} \tilde{\boldsymbol{\sigma}}\right\|_{L_{y^{-\alpha}}^{2}\left(\Omega \times\left[0, y_{1}\right]\right)}^{2}+h^{2} L^{2-\beta}\left\|\frac{\partial \tilde{\boldsymbol{\sigma}}}{\partial y}\right\|_{L_{y^{-\alpha+\beta}}^{2}\left(\Omega \times\left[0, y_{1}\right]\right)}^{2}\right\} .
\end{aligned}
$$

Analogously, applying now (4.10), we have

$$
\begin{aligned}
& \left\|\sigma_{n+1}-\Pi_{h, n+1} \sigma_{n+1}\right\|_{L_{y^{-\alpha}}^{2}\left(\Omega \times\left[0, y_{1}\right]\right)}^{2} \\
& \quad \leq C\left\{h^{2}\left\|\nabla_{x} \sigma_{n+1}\right\|_{L_{y^{-\alpha}}^{2}\left(\Omega \times\left[0, y_{1}\right]\right)}^{2}+y_{1}^{2}\left\|\frac{\partial \sigma_{n+1}}{\partial y}\right\|_{L_{y^{-\alpha}}^{2}\left(\Omega \times\left[0, y_{1}\right]\right)}^{2}\right\},
\end{aligned}
$$

and therefore, using again the definition of $y_{1}$, we obtain

$$
\begin{aligned}
& \left\|\sigma_{n+1}-\Pi_{h, n+1} \sigma_{n+1}\right\|_{L_{y^{-\alpha}}^{2}\left(\Omega \times\left[0, y_{1}\right]\right)}^{2} \\
& \quad \leq C\left\{h^{2}\left\|\nabla_{x} \sigma_{n+1}\right\|_{L_{y^{-\alpha}}^{2}\left(\Omega \times\left[0, y_{1}\right]\right)}^{2}+h^{2} L^{2}\left\|\frac{\partial \sigma_{n+1}}{\partial y}\right\|_{L_{y-\alpha}^{2}\left(\Omega \times\left[0, y_{1}\right]\right)}^{2}\right\} .
\end{aligned}
$$

Consequently, combining the estimates above, we conclude

$$
\begin{array}{r}
\left\|\boldsymbol{\sigma}-\Pi_{h} \boldsymbol{\sigma}\right\|_{L_{y^{-\alpha}}^{2}\left(\Omega \times\left[0, y_{1}\right]\right)}^{2} \\
\leq C\left\{h^{2}\left\|D_{x} \boldsymbol{\sigma}\right\|_{L_{y^{-\alpha}}^{2}\left(\Omega \times\left[0, y_{1}\right]\right)}^{2}+h^{2} L^{2-\beta}\left\|\frac{\partial \tilde{\boldsymbol{\sigma}}}{\partial y}\right\|_{L_{y^{-\alpha+\beta}}^{2}\left(\Omega \times\left[0, y_{1}\right]\right)}^{2}\right. \\
\left.+h^{2} L^{2}\left\|\frac{\partial \sigma_{n+1}}{\partial y}\right\|_{L_{y^{-\alpha}}^{2}\left(\Omega \times\left[0, y_{1}\right]\right)}^{2}\right\} .
\end{array}
$$

Applying now (4.9) and (4.10) for the elements of the form $P=K \times\left[y_{j}, y_{j+1}\right]$, for each $j \geq 1$, and summing over these elements we obtain

$$
\begin{aligned}
\| \boldsymbol{\sigma}- & \Pi_{h} \boldsymbol{\sigma} \|_{L^{2}-\alpha}^{2}\left(\Omega \times\left[y_{j}, y_{j+1}\right]\right) \\
& \leq C\left\{h^{2}\left\|D_{x} \boldsymbol{\sigma}\right\|_{L_{y^{-\alpha}}^{2}\left(\Omega \times\left[y_{j}, y_{j+1}\right]\right)}^{2}+\left(y_{j+1}-y_{j}\right)^{2}\left\|\frac{\partial \boldsymbol{\sigma}}{\partial y}\right\|_{L_{y^{-\alpha}}^{2}\left(\Omega \times\left[y_{j}, y_{j+1}\right]\right)}^{2}\right\},
\end{aligned}
$$

and using (5.19),

$$
\begin{aligned}
\| \boldsymbol{\sigma}- & \Pi_{h} \boldsymbol{\sigma} \|_{L^{2}-\alpha}^{2}\left(\Omega \times\left[y_{j}, y_{j+1}\right]\right) \\
\leq & C\left\{h^{2}\left\|D_{x} \boldsymbol{\sigma}\right\|_{L_{y^{-\alpha}}^{2}\left(\Omega \times\left[y_{j}, y_{j+1}\right]\right)}^{2}+C_{\beta} h^{2} y_{j}^{\beta} L^{2-\beta}\left\|\frac{\partial \boldsymbol{\sigma}}{\partial y}\right\|_{L_{y-\alpha}^{2}\left(\Omega \times\left[y_{j}, y_{j+1}\right]\right)}^{2}\right\} \\
\leq & C\left\{h^{2}\left\|D_{x} \boldsymbol{\sigma}\right\|_{L_{y^{-\alpha}}^{2}\left(\Omega \times\left[y_{j}, y_{j+1}\right]\right)}^{2}+C_{\beta} h^{2} L^{2-\beta}\left\|\frac{\partial \boldsymbol{\sigma}}{\partial y}\right\|_{L_{y^{-\alpha+\beta}}^{2}\left(\Omega \times\left[y_{j}, y_{j+1}\right]\right)}^{2}\right\},
\end{aligned}
$$


and then, observing that

$$
L^{2-\beta}\left\|\frac{\partial \sigma_{n+1}}{\partial y}\right\|_{L_{y^{-\alpha+\beta}}^{2}\left(\Omega \times\left[y_{j}, y_{j+1}\right]\right)}^{2} \leq L^{2}\left\|\frac{\partial \sigma_{n+1}}{\partial y}\right\|_{L_{y^{-\alpha}}^{2}\left(\Omega \times\left[y_{j}, y_{j+1}\right]\right)}^{2},
$$

summing over $j$, and combining this with (5.23), we obtain

$$
\begin{array}{r}
\left\|\boldsymbol{\sigma}-\Pi_{h} \boldsymbol{\sigma}\right\|_{L^{2}{ }^{2-\alpha}}^{2}\left(\mathcal{C}_{L}\right) \\
\leq C\left\{h^{2}\left\|D_{x} \boldsymbol{\sigma}\right\|_{L_{y^{-\alpha}}^{2}\left(\mathcal{C}_{L}\right)}^{2}+h^{2} L^{2-\beta}\left\|\frac{\partial \tilde{\boldsymbol{\sigma}}}{\partial y}\right\|_{L_{y-\alpha+\beta}^{2}\left(\mathcal{C}_{L}\right)}^{2}\right. \\
\left.+h^{2} L^{2}\left\|\frac{\partial \sigma_{n+1}}{\partial y}\right\|_{L_{y^{-\alpha}}^{2}\left(\mathcal{C}_{L}\right)}^{2}\right\},
\end{array}
$$

where, here and in what follows, the constant $C$ depends on $C_{\beta}$.

Applying now Lemma 5.1 and the bound (5.24) it follows from (5.22) that

$$
\left\|\boldsymbol{\sigma}-\boldsymbol{\sigma}_{L, h}\right\|_{L_{y^{-\alpha}}^{2}\left(\mathcal{C}_{L}\right)} \leq C h L\|f\|_{\mathbb{H}^{1-s}(\Omega)}+C e^{-\sqrt{\lambda_{1}} L / 4}\|f\|_{\mathbb{H}^{-s}(\Omega)} .
$$

From the hypothesis on $C_{1}$ we have $e^{-\sqrt{\lambda_{1}} L / 4} \leq h$ and, therefore, (5.20) is proved.

In view of (5.15), to finish the proof of (5.21) it is enough to show that

$$
\left\|u-P_{h} u\right\|_{L_{y^{\alpha}}^{2}\left(\mathcal{C}_{L}\right)} \leq C h L\|f\|_{\mathbb{H}^{-s}(\Omega)} .
$$

Using (4.7) for elements of the form $K \times\left[0, y_{1}\right]$ we obtain

$$
\begin{aligned}
\| u- & P_{h} u \|_{L_{y^{\alpha}}^{2}\left(\Omega \times\left[0, y_{1}\right]\right)} \\
& \leq C\left\{h^{\frac{2}{2-\beta}} L\left\|\frac{\partial u}{\partial y}\right\|_{L_{y^{\alpha}}^{2}\left(\Omega \times\left[0, y_{1}\right]\right)}+h\left\|\nabla_{x} u\right\|_{L_{y^{\alpha}}^{2}\left(\Omega \times\left[0, y_{1}\right]\right)}\right\} \\
& \leq C h L\|\nabla u\|_{L_{y^{\alpha}}^{2}\left(\Omega \times\left[0, y_{1}\right]\right)},
\end{aligned}
$$

because $2 /(2-\beta) \geq 1$ and $L \geq 1$.

On the other hand, (4.7) and (5.19) yields

$$
\left\|u-P_{h} u\right\|_{L_{y^{\alpha}}^{2}\left(\Omega \times\left[y_{1}, L\right]\right)} \leq C h L\|\nabla u\|_{L_{y^{\alpha}}^{2}\left(\Omega \times\left[y_{1}, L\right]\right)}
$$

and, therefore, taking into account (5.4) and (5.25) is proved.

\section{Numerical Results}

Now we give some examples showing the asymptotic behavior of the error proved in Theorem 5.3. We solve Problem (5.2) with $\Omega=(0,1) \times(0,1)$ and

$$
f\left(x_{1}, x_{2}\right)=\left(2 \pi^{2}\right)^{s} \sin \left(\pi x_{1}\right) \sin \left(\pi x_{2}\right) .
$$

Recall that $0<s<1$ and $\alpha=1-2 s$. In this case the solution is given by

$$
u\left(x_{1}, x_{2}, y\right)=\frac{2^{1-s}}{\Gamma(s)}(\sqrt{2} \pi y)^{s} K_{s}(\sqrt{2} \pi y) \sin \left(\pi x_{1}\right) \sin \left(\pi x_{2}\right)
$$



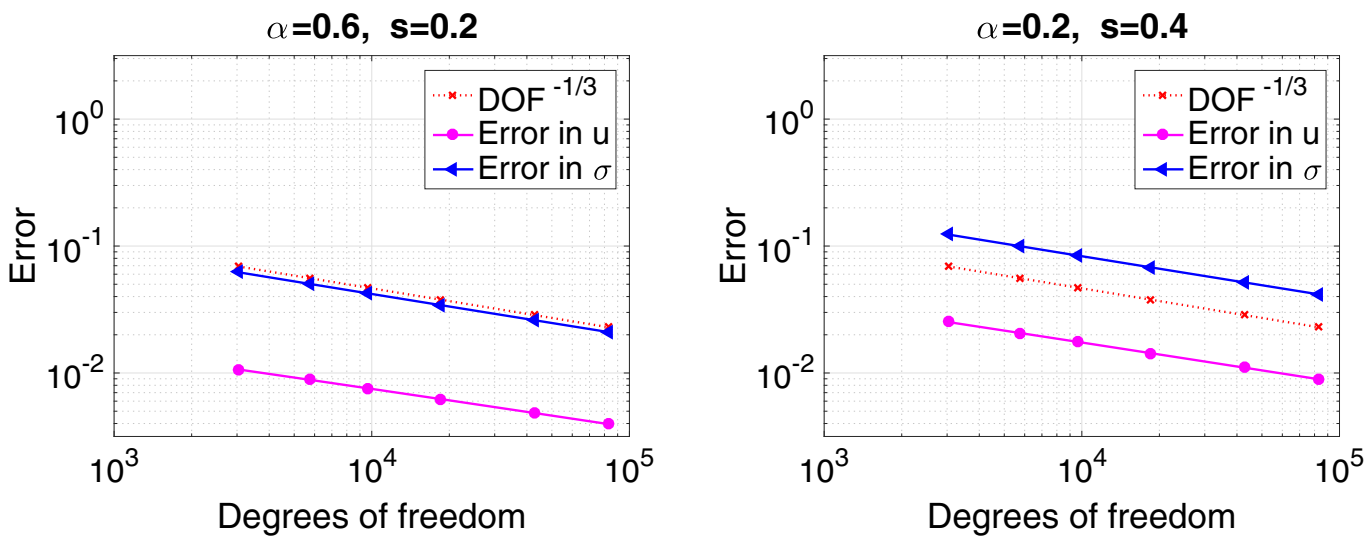

FiguRE 1. Rate of convergence: left $\alpha=0.6$, right $\alpha=0.2$.
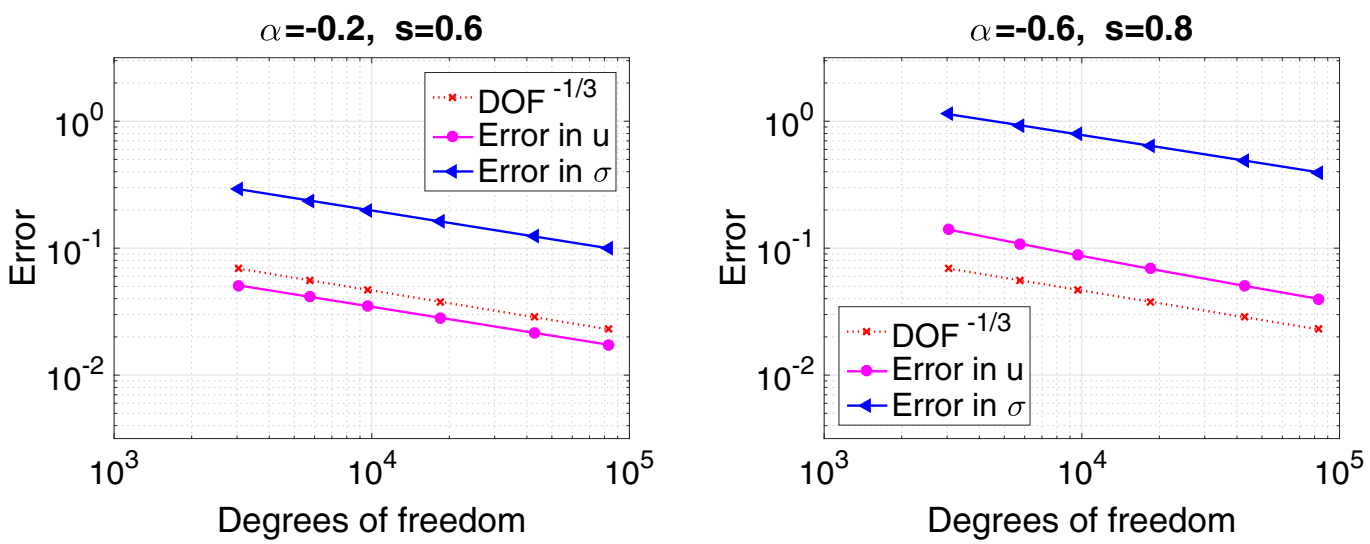

FiguRE 2. Rate of convergence: left $\alpha=-0.2$, right $\alpha=-0.6$.

where $K_{s}$ is a modified Bessel function of the second kind (see [30]). We have used Octave for the numerical integrations and to solve the discrete systems.

We use prismatic elements given by a uniform mesh of triangles in $\Omega$ and the refinement given by (5.18) in the $y$-direction. Observe that for these meshes $h \sim(\mathrm{DOF})^{-1 / 3}$ where DOF denotes the degrees of freedom. Moreover, we choose $L$ as in Theorem 5.3 with $C_{1}=1$, i.e. $L=|\log h|$.

Figures 1 and 2 show the order of the errors $\left\|\boldsymbol{\sigma}-\boldsymbol{\sigma}_{L, h}\right\|_{L_{y}^{2}-\alpha}\left(\mathcal{C}_{L}\right)$ and $\left\|u-u_{L, h}\right\|_{L_{y^{\alpha}}^{2}\left(\mathcal{C}_{L}\right)}$ for several values of $\alpha$.

\subsection{Postprocess to approximate $v(x)=u(x, 0)$}

Finally, to solve (5.1), we need to approximate $u(x, 0)$ where $u$ is the solution of $(5.2)$. We will use the approximations $u_{L, h}$ and $\boldsymbol{\sigma}_{L, h}$ obtained above.

Since $u_{L, h}$ is only an approximation in the $L^{2}$-norm, one cannot expect that its restriction to $y=0$ be a good approximation of $u(x, 0)$. In order to obtain a better approximation we will make a local correction of $u_{L, h}$ using also the computed $\boldsymbol{\sigma}_{L, h}$. This correction corresponds to a first order Taylor expansion, indeed, the 
formula that we are going to prove in the next lemma is motivated by

$$
u(x, 0) \sim u\left(x, \frac{y_{1}}{2}\right)-\frac{y_{1}}{2} \frac{\partial u}{\partial y}\left(x, \frac{y_{1}}{2}\right) .
$$

We will prove that in this way we obtain an approximation in $L^{2}(\Omega)$ of at least the same order than the mixed finite element approximation of (5.2).

Given $x \in \Omega$ and $0<j<N$ we introduce the jumps

$$
\left[u_{L, h}(x)\right]_{j}=u_{L, h}\left(x, y_{j}^{+}\right)-u_{L, h}\left(x, y_{j}^{-}\right) .
$$

If $x$ is not in the interior of an element $K$ in the partition of $\Omega$ we choose arbitrary an element containing it to evaluate $u_{L, h}$ (this is irrelevant because afterwards we are going to integrate in $x$ ).

We will use the standard piecewise linear basis functions, namely, for $1 \leq j \leq N-1$,

$$
\begin{aligned}
& \tau_{j}(y)=\left\{\begin{array}{llc}
\frac{y_{j+1}-y}{y_{j+1}-y_{j}} & \text { if } & y_{j}<y<y_{j+1} \\
\frac{y-y_{j-1}}{y_{j}-y_{j-1}} & \text { if } & y_{j-1}<y<y_{j},
\end{array}\right. \\
& \tau_{0}(y)=\frac{y_{1}-y}{y_{1}} \quad \text { if } \quad 0<y<y_{1},
\end{aligned}
$$

and

$$
\tau_{N}=\frac{y-y_{N-1}}{y_{N}-y_{N-1}} \quad \text { if } \quad y_{n-1}<y<y_{N}
$$

Lemma 6.1. For any $x \in \Omega$ we have

$$
u_{L, h}(x, 0)+\int_{0}^{L} \tau_{0}(y) y^{-\alpha} \sigma_{L, h, n+1}(x, y) \mathrm{d} y=\int_{0}^{L} y^{-\alpha} \sigma_{L, h, n+1}(x, y) \mathrm{d} y .
$$

Proof. Since $u_{L, h}$ is piecewise constant one can see that

$$
u_{L, h}(x, L)=\sum_{j=1}^{N-1}\left[u_{L, h}(x)\right]_{j}+u_{L, h}(x, 0) .
$$

Let $K$ be the element containing $x$. For $1 \leq j \leq N-1$, taking the function $\left(\mathbf{0}, \tau_{j}\right)$ supported in $K \times\left[y_{j-1}, y_{j+1}\right]$ as test function in (5.13), we have

$$
\int_{0}^{L} \int_{K} y^{-\alpha} \sigma_{L, h} \cdot\left(\mathbf{0}, \tau_{j}\right) \mathrm{d} x \mathrm{~d} y-\int_{0}^{L} \int_{K} u_{L, h} \operatorname{div}\left(\mathbf{0}, \tau_{j}\right) \mathrm{d} x \mathrm{~d} y=0
$$

and, since $\sigma_{L, h, n+1}(x, y)$ is independent of $x$ for $x \in K$, we obtain

$$
\left[u_{L, h}(x)\right]_{j}+\int_{0}^{L} y^{-\alpha} \sigma_{L, h, n+1}(x, y) \tau_{j}(y) \mathrm{d} y=0 .
$$

Analogously, using now $\left(0, \tau_{N}\right)$ yields

$$
u_{L, h}(x, L)=\int_{0}^{L} y^{-\alpha} \sigma_{L, h, n+1}(x, y) \tau_{N}(y) \mathrm{d} y .
$$

Therefore, replacing in (6.2) we have

$$
\sum_{j=1}^{N} \int_{0}^{L} y^{-\alpha} \sigma_{L, h, n+1}(x, y) \tau_{j}(y) \mathrm{d} y=u_{L, h}(x, 0)
$$

which immediately gives (6.1) because $\sum_{j=0}^{N} \tau_{j} \equiv 1$. 
To approximate the solution of (5.1) given by $v(x)=u(x, 0)$ we introduce

$$
v_{L, h}(x)=u_{L, h}(x, 0)+\int_{0}^{L} \tau_{0}(y) y^{-\alpha} \sigma_{L, h, n+1}(x, y) \mathrm{d} y .
$$

We also define $v_{L}(x)=u_{L}(x, 0)$.

\section{Lemma 6.2.}

$$
\left\|v_{L}-v_{L, h}\right\|_{L^{2}(\Omega)} \leq \frac{1}{\sqrt{1-\alpha}} L^{\frac{1-\alpha}{2}}\left\|\boldsymbol{\sigma}-\boldsymbol{\sigma}_{L, h}\right\|_{L_{y^{-\alpha}}^{2}\left(\mathcal{C}_{L}\right)} .
$$

Proof. Since $u_{L}(x, L)=0$ and, recalling that $\frac{\partial u_{L}}{\partial y}=-y^{-\alpha} \sigma_{n+1}$, we have

$$
v_{L}(x)=\int_{0}^{L} y^{-\alpha} \sigma_{n+1}(x, y) \mathrm{d} y .
$$

Therefore, using (6.1) and the definition of $v_{L, h}$, we obtain

$$
v_{L}(x)-v_{L, h}(x)=\int_{0}^{L} y^{-\alpha}\left(\sigma_{n+1}(x, y)-\sigma_{L, h, n+1}(x, y)\right) \mathrm{d} y,
$$

and, applying the Schwarz inequality,

$$
\left|v_{L}(x)-v_{L, h}(x)\right|^{2} \leq\left(\int_{0}^{L} y^{-\alpha} \mathrm{d} y\right) \int_{0}^{L} y^{-\alpha}\left|\left(\sigma_{n+1}(x, y)-\sigma_{L, h, n+1}(x, y)\right)\right|^{2} \mathrm{~d} y,
$$

and integrating now in $x$ we conclude the proof.

We can now prove the error estimate for the approximation of the solution of the fractional Laplacian.

Theorem 6.3. Under the hypotheses of Theorem 5.3 we have

$$
\left\|v-v_{L, h}\right\|_{L^{2}(\Omega)} \leq C h|\log h|^{\frac{3-\alpha}{2}}\|f\|_{\mathbb{H}^{1-s}(\Omega)},
$$

where the constant is as in Theorem 5.3 an depends also on $\alpha$.

Proof. From Lemma 6.2 and, recalling that $L=C_{1}|\log h|$, we have

$$
\left\|v_{L}-v_{L, h}\right\|_{L^{2}(\Omega)} \leq C|\log h|^{\frac{1-\alpha}{2}}\left\|\boldsymbol{\sigma}-\boldsymbol{\sigma}_{L, h}\right\|_{L^{2}-\alpha}\left(\mathcal{C}_{L}\right)
$$

where the constant depends on $\alpha$. Combining this estimate with (5.20) we obtain

$$
\left\|v_{L}-v_{L, h}\right\|_{L^{2}(\Omega)} \leq C h|\log h|^{\frac{3-\alpha}{2}}\|f\|_{\mathbb{H}^{1-s}(\Omega)} .
$$

It remains to estimate $v-v_{L}$. But, from the trace theorem given in Proposition 2.5 of [30] combined with (5.11)

$$
\left\|v-v_{L}\right\|_{L^{2}(\Omega)} \leq C\left\|u-u_{L}\right\|_{H_{y^{\alpha}}^{1}(\mathcal{C})} \leq C e^{-\sqrt{\lambda_{1}} L / 4}\|f\|_{\mathbb{H}^{-s}(\Omega)}
$$

and, from the definition of $L$ and $C_{1}$, we obtain

$$
\left\|v-v_{L}\right\|_{L^{2}(\Omega)} \leq C h\|f\|_{\mathbb{H}^{-s}(\Omega)}
$$

which combined with (6.3) concludes the proof. 

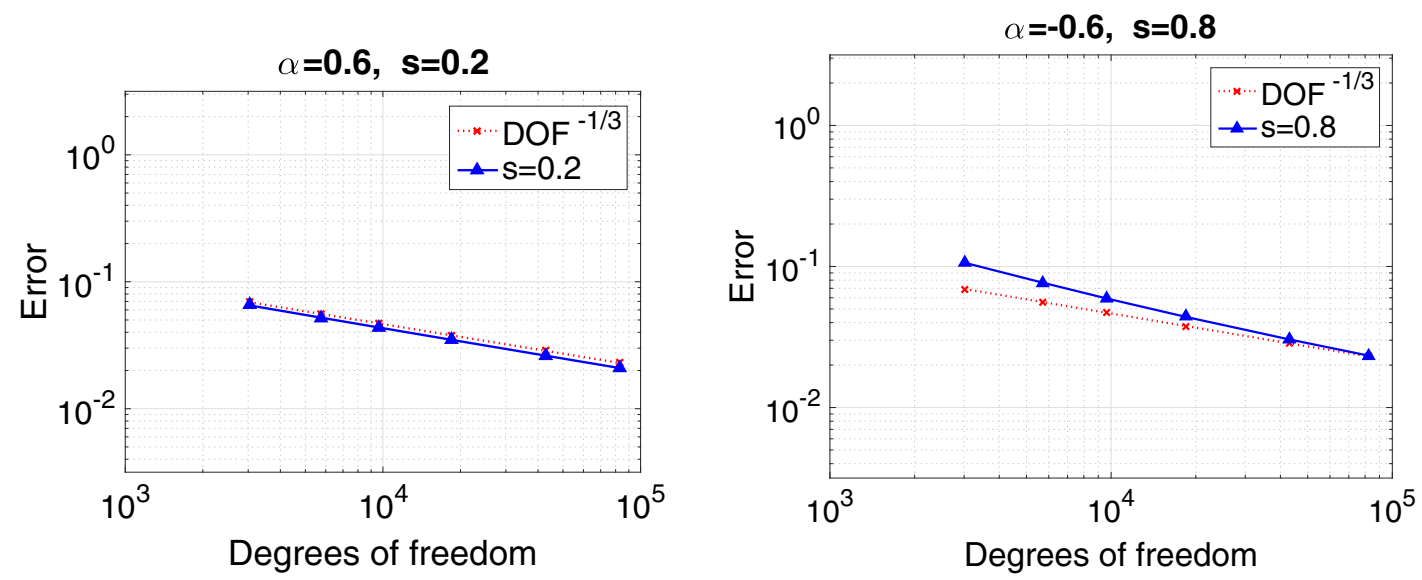

FIGURE 3. Rate of convergence: left $s=0.2$, right $s=0.8$.

Figure 3 shows the order of the error $\left\|v-v_{L, h}\right\|_{L^{2}(\Omega)}$ for problem (5.1) with

$$
f\left(x_{1}, x_{2}\right)=\left(2 \pi^{2}\right)^{s} \sin \left(\pi x_{1}\right) \sin \left(\pi x_{2}\right),
$$

which has as exact solution

$$
v\left(x_{1}, x_{2}\right)=\sin \left(\pi x_{1}\right) \sin \left(\pi x_{2}\right)
$$

Remark 6.4. The order of the error for the approximation of $v$ in the $L^{2}$-norm is probably not the optimal possible. Indeed, with a more complicated postprocessing one could approximate the solution $u$ of problem (5.2) with order almost $O(h)$ in $H_{y^{\alpha}}^{1}(\mathcal{C})$ and, by the trace theorem $\|v\|_{\mathbb{H}^{s}(\Omega)} \leq C\|v\|_{H_{y^{\alpha}}^{1}(\mathcal{C})}$ proved in Proposition 2.5 of [30], one would have the same order for the approximation of $v$ in the $\mathbb{H}^{s}$-norm. Therefore, it is reasonable to expect a higher order in $L^{2}$. This problem requires a different analysis and will be the object of our further research. Let us mention also that such a higher order error estimate has been proved in Proposition 4.7 of [32] for the standard finite element method analyzed in [30].

Acknowledgements. We thank Enrique Otárola for helpful comments. Supported by ANPCyT under grant PICT 2014-1771, by CONICET under grant 11220130100006CO and by Universidad de Buenos Aires under grant 20020120100050 BA.

\section{REFERENCES}

[1] G. Acosta and R.G. Durán, The maximum angle condition for mixed and nonconforming elements: application to the Stokes equations. SIAM J. Numer. Anal. 37 (1999) 18-36.

[2] G. Acosta and R.G. Durán, Divergence Operator and Related Inequalities. Springer Briefs in Mathematics. Springer, New York (2017).

[3] G. Acosta, R.G. Durán and M.A. Muschietti, Solutions of the divergence operator on John domains. Adv. Math. 206 (2006) 373-401.

[4] J.P. Agnelli, E.M. Garau and P. Morin, A posteriori error estimates for elliptic problems with Dirac measure terms in weighted spaces. ESAIM: M2AN 48 (2014) 1557-1581.

[5] T. Arbogast and A.L. Taicher, A linear degenerate elliptic equation arising from two-phase mixtures. SIAM J. Numer. Anal. 54 (2016) 3105-3122.

[6] D.N. Arnold, D. Boffi and R.S. Falk, Quadrilateral H(div) finite elements. SIAM J. Numer. Anal. 42 (2005) $2429-2451$. 
[7] D. Boffi, F. Brezzi, L.F. Demkowicz, R.G. Durán, R.S. Falk and M. Fortin, Mixed Finite Elements, Compatibility Conditions, and Applications. Lectures given at the C.I.M.E. Summer School held in Cetraro, June 26-July 1, 2006, edited by B. and L. Gastaldi In: Vol. 1939 of Lecture Notes in Mathematics. Springer, Berlin-Heidelberg.

[8] D. Boffi, F. Brezzi and M. Fortin, Mixed Finite Element Methods and Applications. In: Vol. 44 of Springer Series in Computational Mathematics. Springer, Berlin-Heidelberg (2013).

[9] S.C. Brenner and L.R. Scott, The Mathematical Theory of Finite Element Methods, 3rd edition. In: Vol. 15 of Texts in Applied Mathematics. Springer, New York (2008).

[10] L. Caffarelli and L. Silvestre, An extension problem related to the fractional Laplacian. Commun. Part. Differ. Equ. 32 (2007) $1245-1260$.

[11] S.K. Chua, Weighted Sobolev inequalities on domains satisfying the chain condition. Proc. Am. Math. Soc. 117 (1993) $449-457$.

[12] R.R. Coifman and C. Fefferman, Weighted norm inequalities for maximal functions and singular integrals. Stud. Math. 51 (1974) 241-250.

[13] L. Diening, M. Ružička and K. Schumacher, A decomposition technique for John domains. Ann. Acad. Sci. Fenn. Math. 35 (2010) $87-114$.

[14] I. Drelichman and R.G. Durán, Improved Poincaré inequalities with weights. J. Math. Anal. App. 347 (2008) $286-293$.

[15] J. Duoandikoetxea, Fourier Analysis. In: Vol. 29 of Graduate Studies in Mathematics. American Mathematical Society, Providence, RI (2001).

[16] T. Dupont and L.R. Scott, Polynomial approximation of functions in Sobolev spaces. Math. Comput. 34 (1980) $441-463$.

[17] R.G. Durán and F. López García, Solutions of the divergence and Korn inequalities on domains with an external cusp. Ann. Acad. Sci. Fenn. Math. 35 (2010) 421-438.

[18] R.G. Durán, A.L. Lombardi and M.I. Prieto, Supercloseness on graded meshes for $Q_{1}$ finite element approximation of a reaction-diffusion equation. J. Comput. Appl. Math. 242 (2013) 232-247.

[19] E.B. Fabes, C.E. Kenig and R.P. Serapioni, The local regularity of solutions of degenerate elliptic equations. Commun. Part. Differ. Equ. 7 (1982) 77-116.

[20] R.S. Falk and J.E. Osborn, Remarks on mixed finite element methods for problems with rough coefficients. Math. Comput. 62 (1994) 1-19.

[21] J.L. García-Cuerva and J.L. Rubio de Francia, Weighted Norm Inequalities and Related Topics. In: Vol. 116 of North-Holland Mathematics Studies. North-Holland Publishing Co. (1985).

[22] V. Girault and P.A. Raviart, Finite Element Methods for Navier-Stokes Equations, Theory and Algorithms. In: Vol. 5 of Springer Series in Computational Mathematics Springer, Berlin-Heidelberg (1986).

[23] R. Hurri-Syrjänen, An improved Poincaré inequality. Proc. Am. Math. Soc. 120 (1994) 213-222.

[24] R. Hurri-Syrjänen, A weighted Poincaré inequality with a doubling weight. Proc. Am. Math. Soc. 126 (1998) 545-552.

[25] T. Kilpeläinen, Weighted Sobolev spaces and capacity. Ann. Acad. Sci. Fenn. Math. 19 (1994) 95-113.

[26] D.S. Kurtz, Littlewood-Paley and multiplier theorems on weighted $L^{p}$ spaces. Trans. Am. Math. Soc. 259 (1980) $235-254$.

[27] A. Lunardi, Interpolation Theory, 3rd edition [of MR2523200]. In: Vol. 16 of Appunti. Scuola Normale Superiore di Pisa (Nuova Serie) [Lecture Notes. Scuola Normale Superiore di Pisa (New Series)]. Edizioni della Normale, Pisa (2018).

[28] L.D. Marini and P. Pietra, Mixed finite element approximation of a degenerate elliptic problem. Numer. Math. 71 (1995) $225-236$.

[29] B. Muckenhoupt, Weighted norm inequalities for the Hardy maximal function. Trans. Am. Math. Soc. 165 (1972) $207-226$.

[30] R.H. Nochetto, E. Otárola and A.J. Salgado, A PDE approach to fractional diffusion in general domains: a priori error analysis. Found. Comput. Math. 15 (2015) 733-791.

[31] R.H. Nochetto, E. Otárola and A.J. Salgado, Piecewise polynomial interpolation in Muckenhoupt weighted Sobolev spaces and applications. Numer. Math. 132 (2016) 85-130.

[32] R.H. Nochetto, E. Otárola and A.J. Salgado, A PDE approach to space-time fractional parabolic problems. SIAM J. Numer. Anal. 54 (2016) 848-873.

[33] K. Schumacher, Solutions to the equation $\operatorname{div} u=f$ in weighted Sobolev spaces. In: Vol. 81 of Parabolic and Navier-Stokes Equations. Part 2, Banach Center Publications. Polish Academy of Sciences, Institute of Mathematics, Warsaw (2008) pp. $433-440$. 